\title{
URSI National Committee Report, XIV General Assembly, Tokyo, September 1963: Commission 2. Tropospheric Radio Propagation
}

This report summarizes the work of Commission 2 since the last General Assembly and is arranged in accordance with the topics to be discussed at the XIVth General Assembly, Tokyo, 1963. References pertinent to each topic are given after the text of the topic and consist mainly of papers published since the last General Assembly; in some cases references are made to reports not generally published and to papers presented at URSI meetings.

Coordinating, organizing, and editing of the report were accomplished under the direction of A. B. Crawford. The following members of Commission 2 had primary responsibilities for preparing the various sections:

R. Bolgiano, Jr., Section 1.

B. R. Bean, Section 2.
S. G. Bigler, Section 3.

D. C. Hogg, Section 4.

A. W. Straiton, Section 5.

J. B. Smyth, Section 6.

J. H. Chisholm and G. H. Pettengill, Section 7.

Other members participating in the preparation of the report include: A. T. Waterman, Jr., U.S. Chairman, Commission 2; W. S. Ament, A. P. Barsis, K. Bullington, T. J. Carroll, J. V. Evans, J. R. Gerhardt, E. E. Gossard, I. Katz, R. S. Kirby, A. H. LaGrone, F. C. Macdonald, R. K. Moore, K. A. Norton, T. F. Rogers, M. C. Thompson, Jr., and J. R. Wait.

Also contributing were the following nonmembers: W. R. Mliff, G. R. Marner, and W. W. Koepsel.

\section{Models of the Troposphere}

Recent work, leading to a fuller understanding of the troposphere and its influence on radio propagation, may be categorized into three broad areas distinguished by the scale of the atmospheric structure involved. These are (1) synoptic-scale phenomena, for which the dominant feature is the horizontal stratification of the medium, (2) smallscale, three-dimensional phenomena, characterized by refractivity irregularities varying in size from kilometers down to centimeters, and (3) micro-scale phenomena that arise in the molecular and atomic nature of the atmosphere.

\subsection{Stratified Structure}

Extensive meteorological investigations have been conducted, and are planned [Petterssen, 1962], to elucidate the ways in which the atmosphere functions and, in particular, the processes responsible for the structure of the troposphere. For example, an analysis of the exchange between kinetic and potential energy on global scales [Saltzman and Fleisher, $1960 ; 1961]$ has indicated that it is primarily the large-scale eddy motions, rather than the zonally averaged motions, which grow directly at the expense of available potential energy. Another interesting study [Charney and Drazin, 1961] has shown, by analogy with the transmission of electromagnetic radiation in heterogeneous media, that large-scale eddy energy is normally trapped in the troposphere but may escape to the mesosphere, or tunnel through the reflecting barrier at the equinoxes when the upper-atmosphere mean zonal wind is reversing. At such times vertical eddy transport of heat and momentum may produce a significant modification in the basic atmospheric structure.

On a somewhat smaller scale, considerable attention has been given to the wind profile near the surface under neutral [Blackadar, 1962] and nonneutral [Elliott, 1960; Sellers, 1962] conditions. By relating the exchange coefficient to the rate of dissipation of turbulent energy, and by assuming that the size of the eddies becomes independent of height at a relatively low level, reasonable agreement has been obtained between predicted and observed values of surface stress, surface wind direction, and vertical distribution of exchange coefficient in a neutral atmosphere.

In more direct relation to radio propagation phenomena the distribution of microwave refractive index associated with a continental cold outbreak has been studied [Jehn, 1961]. This has led to the suggestion that the techniques of quantitative synoptic climatology may be usefully applied to radio meteorological problems.

The vertical pronile of radio refractive index has been modeled extensively [Bean, 1961a; Martin and Waldron, 1961], and some measurements indidate close agreement with an exponential form [Sherr, 1961; Anderson, Beyers, and Rainey, 1960; Bean, 1961b]. A discussion of the validity and relative merits of the simple exponential profile [Misme, 
Bean, and Thayer, 1960] has helped clarify the application of these models. Bean [1961a] proposed a bi-exponential model for the profile that distinguishes the scale-heights of the dry and moist contributions to the index.

The refraction effects that are a consequence of stratification of the refractive index have been considered from several points of view. For "standard" atmospheres, the effective earth radius method has been placed on a firmer foundation by means of conformal mapping [Toman, 1962]. General formulas for the divergence coefficients of the direct and reflected rays in an exponential atmosphere have been developed [Wilkerson, 1962]. Rainey and Thorn [1962] and Cramond and Thorn [1962] have reanalyzed the refraction problem for the general atmosphere, whether spherically symmetric or not. On the basis of a sizable body of radar angle observations, they conclude that azimuthal corrections such as they have proposed would be valuable.

The influence of refraction on transmission loss in beyond-horizon circuits has been investigated. Use of a larger modified earth radius in subtropical regions (a $K$-value of 1.7 over land and 2.4 over water) has been found to bring the corresponding loss data into line with the standard values for temperate climates [Gray, 1961]. Dennis [1962] has discovered that, for coastal and over-water links, refractivity data derived from meteorological parameters ordinarily used in weather forecasting can be used successfully in the short-term prediction of transmission loss. Surface refractivity has been found to be as useful a predictor of diurnal and seasonal variations of monthly median transmission loss, as are actual radio measurements from prior years [Bean, Fehlhaber, and Grosskopf, 1962].

Normal mode theory and internal reflections, as an explanation for transhorizon propagation, have continued to be the focus of considerable controversy. A theory previously developed to account for a single elevated inversion has been extended [Northover, 1961a] to the case of multiple layers. Northover [1961b] has shown that under certain conditions several weak, high-level inversions can produce an effect similar to a single strong inversion. Bullington [1960] has presented a quantitative explanation of tropospheric and ionospheric "scatter" transmission based on diffraction theory and the average decrease in the index of refraction with height above the earth. Although somewhat different in details of analysis, this approach bears strong resemblance to normal mode theory as applied earlier by Carroll and Ring [1955]. Carroll has supported this model at various URSI meetings, where he has offered interpretations of Tukizi's and of Ponomarenko's work and has supported their general results. On the other hand, Tukizi's results have been criticized [Logan, 1962] on the grounds that his application of the "saddle-point" method to the evolution of an integral involving nonisolated saddle points leads to erroneous results.

The role of atmospheric ducts in radio propagation, particularly that of elevated ducts in beyond- horizon transmission, has been investigated extensively. (See section 6, Guided Waves in the Troposphere.)

\section{2. Small-Scale Irregularities}

Nonspherically stratified structure of the atmosphere and its effect on radio propagation have continued to receive a major share of the attention of U.S. workers. Three relatively new facets of this problem have emerged during the past several years and are now recognized as bearing considerable importance on the development of the field. These are the occurrence and influence of internal gravity waves, the anisotropic structure of atmospheric turbulence, and the broad-band communicationtheoretic representation of transhorizon radio circuits. There has been, of course, a great amount of effort devoted to more familiar topies such as isotropic turbulence and refractive-index spectra, electromagnetic theory of scattering, and the relation of transmission loss and other characteristics of received fields to meteorological conditions. Nonetheless, the emphasis placed upon the more recently initiated areas of investigation is increasing.

The possible role of internal atmospheric waves on the propagation of radio signals beyond the horizon was suggested by the results observed in the Stanford University rapid beam-swinging experiments [Waterman, 1959]. Subsequent analyses by Waterman and Strohbehn [1962] and by Gossard [1962a] have delineated this problem in some detail. Waterman believes that partial reflections (single and multiple) and focusing due to layer curvature may occur. He also concludes that velocities of reflecting facets may exceed greatly the normal wind and wave velocities. Gossard [1960a, 1962a] sup. ports the existence of such waves with meteorolog ical evidence and has deduced that they cause angle of-arrival perturbations on microwave links in th troposphere. He has expressed doubts, however as to the occurrence of sufficiently intense gradient to account for the partial reflections and he doe: not find evidence of the velocities proposed by Waterman. On the other hand, Harney [1962] of the USAF Cambridge Research Laboratories explained a case of anomalous returns covering a microwave radar PPI on a cloudless day in terms of multiple reflections from an undulating surface be tween layers of differing index of refraction. Th role of internal gravity waves in conveying energ. out of the troposphere into the mesosphere ani lower ionosphere has also been explored [Gossard $1962 \mathrm{~b}]$. It has been discovered that a window exists at periods of about $10 \mathrm{~min}$ to $2 \mathrm{hr}$ through which fairly large amounts of energy sometimes flow out of the troposphere.

The anisotropic nature of atmospheric turbulence was noted and a possible indirect influence on tropospheric scatter propagation was deduced [Bolgiano, 1960]. The detailed structure of turbulence in a stratified medium has been developed [Deissler, 1962 Bolgiano, 1962], and wind-tunnel experiments hav been performed [Wiskind, 1962] that showed son 
of the effects of stratification. The electromagnetic effects of anisotropic structure have been explored [Gjessing, 1962] by expressing the scattering coefficient in terms of the spectrum of refractive index deviations, which (anisotropic) fluctuations have, in turn, been modeled in Fourier space. The group at Standford University has carried out a simultaneous frequency-scan and rapid beam-swinging experiment, [Waterman, Strohbehn, and Coates, 1960]. The transmitter and receiver have been tuned synchronously over a $20 \mathrm{Mc} / \mathrm{s}$ band at a $300-c y c l e$ rate while the $1 / 2$ deg antenna beam was being swept through a 4 deg azimuth sector at a 10 -cycle rate. Detection of changes in the coherence angle in the absence of corresponding changes in the coherence bandwidth has been interpreted as evidence of variation in the anisotropy of the refractive index structure from time to time. A preliminary experiment in which radio scatter sionals have been employed to measure simultaneously the shape of the refractive index spectrum for vertical and nearly horizontal wavenumber components has been reported by Bolgiano [1962]. In the same range of wavenumber magnitude the vertical spectrum exhibited steeper slope than the near horizontal spectrum on almost all occasions. An extensive, three-dimensional refractive index measurement program has been initiated by Thompson and Janes of the National Bureau of Standards. Multiple sensing cavities will be mounted on a tower, along the mean wind, transverse thereto, and vertically so as to measure instantaneously the three-dimensional correlation function.

Information-theoretic analysis of transhorizon radio circuits has been placed on a firmer basis as a consequence of studies made on the problem of signalling through random channels. (Commission 6) Theoretical work in this area is intimately dependent upon the model of tropospheric structure, in space and time, that one assumes. By careful analysis of measurements of this type it may be possible to deduce important information about the structure. Much effort continues to be applied in this direction.

Chisholm and his colleagues at M.I.T. Lincoln Laboratory have continued the analysis of data gathered during the 1950's. They have found [Chisholm et al., 1962] that at $400 \mathrm{Mc} / \mathrm{s}$ on a $640-\mathrm{mi}$ path the propagation time of pulse transmissions , changes as though the height of the scattering layer were varying between $20,000 \mathrm{ft}$ and $90,000 \mathrm{ft}$. Álso on a similar length path they noted frequency selective fading such that the crosscorrelation was down to 0.7 at $100 \mathrm{kc} / \mathrm{s}$ separation. On a shorter path [Chisholm et al., 1961], carrier-frequency deviations and path-length variations were measured by a round-trip transmission system. Carrierfrequency stability was determined to exceed 1 part in $10^{8}$ and rms path-length fluctuations were found to be about $50 \mathrm{~m}$. Measurements over a 188-mi path at $2290 \mathrm{Mc} / \mathrm{s}$ using $0.1 \mu$ sec pulses were reported by Roche et al. [1962]. A large percentage of the time the pulses were received undistorted.
Use of this very short pulse length provided a means of resolving the microstructure of the atmosphere.

The range of fading (10 to 90 percent) and its correlation with meteorological parameters has been studied [Bauer, 1961]. In particular, strong correlation was observed between this range (12 to $30 \mathrm{db}$ ) and the wind shear near the common volume. The fading range approximated closely the theoretical $13.5 \mathrm{db}$ when the shear was low but increased rapidly with increasing shear. Since the data were normalized to hourly median values, the excess fading range could be accounted for on the basis of large-scale inhomogeneities of the mean square refractiveindex fluctuations. The depth and duration of fades have also been analyzed [Wright and Cole, 1960] for signals transmitted over a $100-\mathrm{mi}$ path at $950 \mathrm{Mc} / \mathrm{s}$. A large percent of fades are of short duration, particularly the deep fades. Of those $25 \mathrm{db}$ or more below the median, only 1 percent were greater than $100 \mathrm{msec}$ in length. Both phase and amplitude fluctuations were measured for $810 \mathrm{Mc} / \mathrm{s}$ signals on a 100-mi path, as related by Beamer [1962] of Collins Radio Company. Of particular interest is the fact that the distribution of phase excursions did not approach the distribution predicted by the multipath scattering model even though the short-term amplitude distribution was closely Rayleigh. Also the observation of rapid changes in phase and amplitude, in short bursts, for a half-hour just prior to sunset, regardless of local weather along the path, requires some explanation.

The use of monopulse radar techniques for the experimental analysis of scattered radio waves has been described by Temple [1962] of M.I.T. Lincoln Laboratory at an U.R.S.I. meeting.

The detail structure of tropospheric properties has been explored extensively, to obtain information essential in the application of various models designed to explain radio propagation, as well as to provide data that are useful in the evaluation of turbulence theory and in the interpretation of such theory in the atmosphere. Spectra of pressure and refractive index fluctuations [Gossard, 1960a, 1960b], derived from data collected in the southern Pacific coastal region of the United States, exhibit two features that are of special interest. First, very high mean square fluctuation intensity is concentrated in relatively thin strata within the lower part of stably stratified layers. It would appear that turbulence in the well-mixed convective region below stirs (or otherwise disturbs) the stratified layer, with its established gradients, thus producing marked deviations of atmospheric properties at the inversion and immediately above, but having little effect at greater altitudes. In these zones of intense refractive index variance the spectra show a well-developed $-5 / 3$ power-law form for horizontal scales of up to 1,000 $\mathrm{ft}$ or more. Second, although outside these zones the spectral intensity shows characteristic departures from this power-law at low wave numbers, corresponding to varying deficits of mean square fluctuation "energy" at the larger scale sizes, at the high wave numbers ( 1 to $100 \mathrm{ft}$ ) the theoretical 
inertial-subrange power-law is very closely satisfied and the intensity is surprisingly constant, independent of stability and height. Similar data from an inland region in the southern United States [Straiton, Deam, and Walker, 1962] indicate a power-law spectral form in which the exponent varies from -1 to -3.3 , the slope decreasing at higher altitudes, and an intensity that varies widely at the smaller scales (less than $20 \mathrm{ft}$ ), generally decreasing with increased height.

Investigation of the refractive index structure by radio-scattering techniques at several frequencies simultaneously has shown, for the Pacific coastal region [Ortwein, Hopkins, and Pohl, 1961], both angle and wavelength dependence that is in agreement with the $-5 / 3$ power-law form for the spectrum. Reflections from layers appear to be important at the lower frequencies (below 1,000 Mc/s) when the atmosphere is characterized by strongly stable regions. Bolgiano [1961] of Cornell reported that similar multiple frequency experiments employing scaled antennas on a hilly, inland path in the northeast United States indicate a highly variable form for the spectrum. The median value of the exponent in the power-law is deduced to be approximately $-5 / 3$ in summer but approaches $-7 / 3$ in winter. Dar to day variation of the deduced exponent ranges from -1 to -3 .

Turbulence and wind structure have been studied by a great many workers, both fluid dynamicists and meteorologists. Reference is made here only to a few investigations that have fairly direct applicability to the radio problem itself or to the modeling of refractive index structure. Turbulence in shear flow [Deissler, 1961] and in the presence of a temperature gradient [Deissler, in press] has been analyzed and it has been shown that the presence of the gradients effects a transfer of fluctuation "energy" from large to small scales. The ratio of eddy conductivity to eddy viscosity in the atmosphere is shown to vary from one to slightly over two, depending upon the intensity of the shear. Kuo [1962] has considered the influence of eddy diffusion on thermal convection, while Ogura [1962a, 1962b] has analyzed buoyant convection in the atmosphere, including the effect of latent heat of vaporization. The relation of Richardson number to potential temperature gradient has been studied [Reed, 1960], with the conclusion that a decrease in temperature gradient does not necessarily imply a decrease in $R_{i}$. Gifford [1961] has reemphasized the importance of relative diffusion as opposed to average dispersion in describing turbulent diffusion in the atmosphere. The role of eddy conductivity in subsidence inversions has been treated by Panofsky [1961].

Frequency spectra of large-scale turbulent transfer of momentum and heat have been calculated from upper air data [Chui, 1960], indicating that the lower frequency components are more important in the northward transport of heat at higher altitudes. Periods in excess of 20 days were found to be significant. Vertical velocity fluctuations have been derived from the Monin-Obukhov similarity hypothesis [Munn, 1961]. The results indicate rms vertical velocities increase with height in superadiabatic conditions, decrease with height in stable conditions, and are independent of height in a neutral atmosphere. L'Hermitte [1962] has measured velocity components by Doppler radar. From these data he has been able to infer the order of magnitude and variance of vertical wind currents. The possibility of extreme turbulence has been investigated on the basis of buoyancy and horizontal divergence in a particular synoptic situation [House and Lee, 1961]. Variation of the rate of dissipation of turbulent energy as a function of altitude has been discussed by Lettau [1961] who points out that estimates of $\mathscr{E}$ vary by three orders of magnitude at any given altitude. In reply Wilkins [1961] notes that the median estimate of $\delta$ decreases about one order of magnitude for each order of magnitude increase in height. Gifford [1962] has concluded that, in spite of some claims to the contrary, estimates of $\mathscr{E}$ based on diffusion analysis are in agreement with those of other methods.

Low-level wind structure has been analyzed by means of constant volume balloon flights [Angell and Pack, 1962]. Evidence was found of gravity wave oscillations. Also horizontal rms velocity components were found to be twice vertical components. Two-point wind correlation tensor is similar to that of homogeneous, isotropic turbulence provided horizontal homogeneity is assumed [Buell, 1960]. Models of vertical correlations of the wind have been constructed from extensive sets of data in the northern hemisphere [Kochanski, 1961]. These show very similar magnitude and shape in large zones of latitude. The correlation runs high in the midtroposphere (7 to $9 \mathrm{~km})$, and the decay of correlation with vertical separation is often nearly exponential. The sea breeze has been studied theoretically by Estoque [1961, 1962], who has calculated the evolution for different large-scale wind and temperature fields.

The stochastic nature of refractive index irregularities has been considered by Bugnolo [1961], who proposes a model that takes account of the nonstationary character of all atmospheric processes. The same author has also investigated the question of multiple scattering in the troposphere [Bugnolo, 1960a] and has derived a transport equation for the spectral density of a multiply-scattered electromagnetic field [Bugnolo, 1960b]. Furutsu [1962] of the National Bureau of Standards has developed a statistical theory of electromagnetic fields in fluctuating media, showing that this problem may be solved by use of the same methods as are employed in quantum field theory. Krzywoblocki [1962] of Michigan State University has proposed a solution to the equation of wave propagation through the irregular troposphere by means of integral operators. The use of agitated styrofoam spheres to simulate a randomly irregular medium and the experimental investigation of microwave propagation through such a medium has continued [Beard, 1962].

An empirical model has been deduced for the spectrum of refractive index fluctuations in the tropo- 
sphere on the basis of transmission loss versus distance data and the assumption of vertical homogeneity of the irregularities [Latorre, 1962]. The Booker-Gordon theory of radio scatter propagation has been questioned [Abraham and Bradshaw, 1961]. Working with a set of previously unanalyzed data it was found that, although correlation of received signal strength with appropriate weather parameters on a whole year cycle was high, short-term correlations vanished. Periodic structure in the mean received signal strength on a scatter path was explained at an U.R.S.I. meeting by White [1962] of Cornell University. He proposed patches of enhanced refractive index fluctuations as a consequence of the formation of buoyant plumes.

The performance of antennas in the reception of scattered radio fields has been considered by several workers. Stein and Johansen [1961] have applied the techniques of matched-filter theory to the problem and have shown that a vast reduction in error rates can be effected for narrow-beam antennas over that which would occur using standard antenna design. Waterman [1961] has clarified the nature of the coupling loss in tropospheric scatter as compared with exospheric scatter and he has discussed the relationship as regards coupling loss between a scatter circuit having narrow-beam antennas at both terminals and one having a narrow-beam antenna at one terminal only [Waterman, 1963]. Carroll and Levatich [1962] noted that degradation of antenna performance may be explained in terms of an angular distribution of incident scattered energy and proposed that this angular spread is an inverse function of path length. The dependence of aperture-tomedium coupling loss on distance has also been discussed by Yeh [1962] and Hogg [1962].

\subsection{Atomic and Molecular Phenomena}

With the ever-increasing sensitivity of equipment, absorption and radiation in the atmosphere have become highly important in imposing the ultimate limit on the performance of many systems. Theoretical and experimental studies of these phenomena which are related to the molecular and atomic nature of the atmosphere are described in section 4 , entitled Tropospheric Propogation Affecting Space Communications.

\section{References}

Abraham, L. G., and J. A. Bradshaw (1961), Weather and reception level on a tropospheric link-Annual and shortterm correlations, J. Res. NBS 65D (Radio Prop.), No. 2, 155 .

Anderson, W. L., N. J. Beyers, and R. J. Rainey (1960), Comparison of experimental with computed tropospheric refraction, IRE Trans. AP-8, 456.

Angell, J. K., D. H. Pack (162), Analysis of low-level constant volume balloon (tetroon) flights from Wallops Island, J. Atmospheric Sci. 19, 87.

Bauer, L. H. (1961), Correlation of wind shear with tropospheric scatter signals, IRE Trans. AP-9, 466.

Veamer, C. M. (1962), Phase and amplitude measurements of an experimental tropospheric scatter circuit, a paper presented at URSI Meeting, Ottawa, October.

Bean, B. R. (1961a), Concerning the bi-exponential nature of the tropospheric radio refractive index, Bei. \& Phys. Atmos. 34, 81 .

Bean, B. R. (1961b) Comparison of observed tropospheric refraction with values computed from the surface refractivity, IRE Trans. AP-9, 415.

Bean, B. R., L. Fehlhaber, and J. Grosskopf (1962), Comparative study of the correlation of seasonal and diurnal cycles of transhorizon radio transmission loss and surface refractivity, J. Res. NBS 66D (Radio Prop.), 593.

Beard, C. I. (1962), Statistics of phase quadrature components of microwave fields transmitted through a random medium, IRE Trans. AP-10, 721.

Blackadar, A. K. (1962), The vertical distribution of wind and turbulent exchange in a neutral atmosphere, J. Geophys. Res. 67, 3095.

Bolgiano, R., Jr. (1960), A theory of wavelength dependence in ultrahigh frequency transhorizon propagation based on meteorological considerations, J. Res. NBS 64D (Radio Prop.), 231.

Bolgiano, R., Jr. (1961), Initial results of a scaled threefrequency transhorizon experiment, a paper presented at URSI meeting, Austin, Tex., October.

Bolgiano, R., Jr. (1962), A preliminary measurement of tropospheric anisotropy, a paper presented at Fall URSI meeting, Ottawa.

Bolgiano, R. (1962), Structure of turbulence in stratified media, J. Geophys. Res. 67, 3015.

Buell, C. E. (1960), The structure of two-point wind correlations in the atmosphere, J. Geophys. Res. 65, 3353.

Bugnolo, D. S. (1960a), On the question of multiple scattering in the troposphere, J. Geophys. Res. $65,879$.

Bugnolo, D. S. (1960b), Transport equation for the spectral density of a multiple-scattered electromagnetic field, J. Appl. Phys. 31, 1176.

Bugnolo, D. S. (1961), Stochastic processes and beyond-thehorizon propagation, IRE Trans. AP-9, 226.

Bullington, K. (1960), A quantitative explanation of tropospheric and ionospheric "scatter" transmission, a paper presented at Fall URSI meeting, Boulder, Colo.

Carroll, T. J., and R. M. Ring (1955), Propagation of short waves in a normally stratified troposphere, Proc. IRE 43, 1384.

Carroll, T. J. (1960), Interpretation of Tukizi's calculations of twilight propagation, a paper presented at Fall URSI meeting, Boulder, Colo.

Carroll, T. J. (1962), Ponomarenko's mode system for twilight propagation, a paper presented at Fall URSI meeting, Ottawa.

Carroll, T. J., and J. L. Levatich (1962), Minimizing antenna gain loss in twilight propagation, a paper presented at URSI meeting, Washington, May.

Charney, J. G., and P. G. Drazin (1961), Propagation of planetary-scale disturbances from the lower into the upper atmosphere, J. Geophys. Res. 66, 83.

Chisholm, J. H., S. J. Goodman, J. M. Kennedy, L. B. Lambert, L. P. Rainville, and J. F. Roche (1961), Frequency variation due to over-the-horizon tropospheric propagation IRE Trans. AP-9, 384 .

Chisholm, J. H., W. E. Morrow, Jr., B. E. Nichols, J. F. Roche, and A. E. Teachman (1962), Properties of 400 $\mathrm{mc} / \mathrm{s}$ long-distance tropospheric circuits, Proc. IRE 50, 2464 .

Chui, Wan-cheng (1960), The spectra of large-scale turbulent transfer of momentum and heat, J. Meteorol. 1\%, 435.

Cramond, W. R., and D. C. Thorn (1962), An approach to azimuth angle refraction correction, a paper presented at Spring URSI meeting, Washington.

Deissler, R. G. (1961), Effects of inhomogeneity and of shear flow in weak turbulent fields, Phys. Fluids 4, 1187.

Deissler, R. G. (1962), Turbulence in the presence of a vertical body force and temperature gradient, J. Geophys. Res. 67, 3049 .

Deissler, R. G., Turbulent heat transfer and temperature fluctuations in a field with uniform velocity and temperature gradients, Intern. J. Heat Mass Transfer (in press). 
Dennis, A. S. (1962), Correlation between hourly median scattered signals and simple refractivity parameters, J. Res. NBS 66D (Radio Prop.), 285.

Elliott, W. P. (1960), An hypothesis for the diabatic mixinglength, J. Meteorol. 17, 681 .

Estoque, M. A. (1961), A theoretical investigation of the sea breeze, Quart. J. Roy. Meteorol. Soc. 87, 136.

Estoque, M. A. (1962), The sea breeze as a function of the prevailing synoptic situation, J. Atmospheric Sci. 19, 244.

Furutsu, K. (1962), On the statistical theory of electromagnetic field in fluctuating medium, a paper presented at URSI meeting, Ottawa, October.

Gifford, F. A., Jr. (1961), Small-scale turbulent diffusion in the atmosphere, Nature (London) 190, 248.

Gifford, F. A., Jr. (1962), The vertical variation of atmospheric eddy energy dissipation, J. Atmospheric Sci. 19, 205.

Gjessing, D. T. (1962), On the scattering of electromagnetic waves by nonisotropic inhomogeneties in the atmosphere, J. Geophys. Res. 6\%, 1017.

Gossard, E. E. (1960a), Spectra of atmospheric scalars, J. Geophys. Res. 65, 3339.

Gossard, E. E. (1960b), Power spectra of temperature, humidity, and refractive index from aircraft and tethered balloon measurements, IRE Trans. AP-8, 186.

Gossard, E. E. (1962a), The reflection of microwaves by a refractive layer perturbed by waves, IRE Trans. AP-10, 317.

Gossard, E. E. (1962b), Vertical flux of energy into the lower ionosphere from internal gravity waves generated in the troposphere, J. Geophys. Res. 6\%, 745.

Gray, R. E. (1961), Tropospheric scatter propagation and meteorological conditions in the Caribbean, IRE Trans. $\mathbf{A P}-\mathbf{9}, 492$.

Harney, P. J. (May 1962), A sample of propagation by reflection, a paper presented at URSI meeting, Washington.

Hogg, D. C. (1962), The aperture-to-medium coupling loss in beyond-the-horizon propagation, and reply by L. P. Yeh, Proc. IRE 50, 1529.

House, D. C., and J. T. Lee (1961), A case study of extreme turbulence possibilities based on considerations of buoyance. and horizontal divergence, Bull. Am. Meteorol. Soc. 42, 175.

Jehn, K. H. (1961), Microwave refractive-index distribution associated with the Central United States cold outbreak, Bull. Am. Meteorol. Soc. 42, 77.

Kochanski, A. (1961), Models of vertical correlation of the wind, J. Meteorol. 18, 151.

Krzywoblocki, M. Z. V. (1962), An approach to solution of equations of wave propagation through troposphere by means of integral operators, a paper presented at URSI meeting, Ottawa, October.

Kuo, H. L. (1962), On the controlling influence of eddy diffusion on thermal convection, J. Atmospheric Sci. 19, 236.

Latorre, V. R. (1962), A new scattering coefficient for tropospheric scatter propagation, IRE Trans. AP-10, 471.

Lettau, H. H. (1961), Dissipation of energy by turbulence, J. Meteorol. 18, 125.

L'Hermitte, R. M. (1962), Note on wind variability with Doppler radar, J. Atmospheric Sci. 19, 343.

Logan, N. A. (1962), Comments on "Diffraction theory of tropospheric propagation near and beyond the radio horizon," IRE Trans. AP-10, 98.

Martin, F. L., and C. G. Waldron (1961), A "layered" exponential model of radar refractivity, J. Geophys. Res. 66, 4129.

Misme, P., B. R. Bean, and G. D. Thayer (1960), Models of the atmospheric radio refractive index, Proc. IRE 48, 1498.

Munn, R. E. (1961), A theoretical expression for the rootmean-square vertical eddy fluctuation $\left(\overline{w^{2}}\right)^{1 / 2}$, J. Meteorol. 18. 38 .

Northover, F. H. (1961a and 1961b), The effect of multiple atmospheric inversions on tropospheric radio propagation, J. Atmospheric Terrest. Phys. 20, 295. Also under same title, J. Res. NBS 65D (Radio Prop.), 385.

Ogura, Y. (1962b), Convection of isolated masses of a buoyant fluid: a numerical calculation, J. Atmospheric Sci. 19, 492.

Ogura, Y., and N. A. Phillips (1962a), Scale analysis of deep and shallow convection in the atmosphere, J. Atmospheric Sci. 19, 173.

Ortwein, N. R., R. V. F. Hopkins, and J. E. Pohl (1961), Properties of tropospheric scattered fields, Proc. IRE 49, 788.

Panofsky, H. A. (1961), Eddy conductivity in subsidence inversions, J. Meteorol. 18, 806.

Petterssen, S. (1962), The atmospheric sciences 1961-1971, NAS-NRC Publication 946.

Rainey, R. J., and D. C. Thorn (1962), A radar refraction correction for symmetric and non-symmetric tropospheric index distributions, a paper presented at Spring URSI meeting, Washington.

Reed, R. J. (1960), Some comments on hodograph analysis as applied to the occurrence of clear air turbulence, J. Meteorol. 1\%, 476.

Roche, J. F., P. J. Kitner, J. H. Chisholm, and L. P. Rainville (1962), Measurements over a 188-mile path at $2298 \mathrm{Mc} / \mathrm{s}$ using 1/10 microsecond pulse transmission, a paper presented at URSI meeting, Ottawa.

Saltzman, B., and A. Fleisher (1960), The modes of release of a vailable potential energy in the atmosphere, J. Geophys. Res. 65, 1215 .

Saltzman, B., and A. Fleisher (1961), Further statistics on the modes of release of available potential energy, J. Geophys. Res. 66, 2271.

Sellers, W. D. (1962), A simplified derivation of the diabatic wind profile, J. Atmospheric Sci. 19, 180.

Sherr, P. E. (1961), Some airborne refractive-index measurements at $150 \mathrm{mb}$, J. Meteorol. 18, 494.

Stein, S., and D. E. Johansen (1961), A theory of antenna performance in scatter-type reception, IRE Trans. AP-9, 304.

Straiton, A. W., A. P. Deam, and G. B. Walker (1962), Spectra of radio refractive index between ground level and 5000 feet above ground, IRE Trans. AP-10, 732.

Temple, E. B. (1962), Monopulse measurements of amplitude and angle-of-arrival scintillations, a paper presented at URSI meeting, Washington, May.

Toman, K. (1962), The effective earth radius obtained through conformal mapping, Proc. IRE 50, 485.

Waterman, A. T., Jr. (1959), Tropospheric motions observed in rapid beam-swinging experiments, IRE Trans. AP-y, 106.

Waterman, A. T., Jr., J. W. Strohbehn, and D. B. Coates (1960), Simultaneous frequency- and angle-scan experiment in transhorizon propagation, a paper presented at URSI Meeting, Boulder, Colo.

Waterman, A. T., Jr. (1961), Antenna-beam configurations in scatter communications, Proc. IRE 49, 1452.

Waterman, A. T., Jr., and J. W. Strohbehn (1962), The Reflection of radio waves from an undulating tropospheric layer, a paper presented at Spring URSI Meeting, Washington.

Waterman, A. T., Jr. (1963), Transhorizon aperture-medium coupling loss, Proc. IEEE 51, No. 1, 221.

White, D. P. (1962), The existence during daytime summer conditions of a unique periodic structure of the mean refractive index and its effect on tropospheric scatter propagation, a paper presented at URSI Meeting, Washington, May.

Wilkerson, R. E. (1962), Defocusing of radio rays by the troposphere, J. Res. NBS 66D (Radio Prop.), 479.

Wilkins, E. M. (1961), Dissipation of energy by turbulencereply, J. Meteorol. 18, 126.

Wiskind, H. K. (1962), A uniform gradient turbulent transport experiment, J. Geophys. Res. 67, 3033.

Wright, K. F., and J. E. Cole (1960), Measured distribution of the duration of fades in tropospheric scatter transmissions, IRE Trans. AP-8, 594

Yeh, L. P. (1962), Experimental aperture medium coupling loss, Proc. IRE 50, 205. 


\section{Radio Climatology}

Progress in radio climatology in the period since the last general assembly has been in the area of techniques of measurements of the radio refractive index and its variation, transmission loss characteristics under various propagation modes and in different climatic regions, and the climatic variation of the bending of radio waves.

Simultaneous measurement of the refractive index, $n$, in terms of

$$
N=(n-1) 10^{6},
$$

by fixed balloon and aircraft-mounted sensors [Gossard, 1960] has indicated the equivalence of the Eulerian space and time spectra, and indicates that atmospheric stability has a pronounced effect upon the variation of turbulent intensity with height.

Studies of the climatic distribution of $N_{s}$ ( $N$ at ground level) have been completed for the United States with tentative estimates of similar values for the world [Bean, Horn, and Ozanich, 1960]. Summaries of the variation of $N_{s}$ and the vertical distribution of $N$ have shown it to be a promising index of climatic variation [Bean and Horn, 1961], as well as a sensitive indicator of meteorological changes about intense synoptic-scale storm systems [Jehn, 1960a, 1960b, 1961]. Application of the powerful analysis methods of classical meteorology, vertical motion, and potential vorticity indicates their eventual use in day-to-day prediction of the extended ranges of VHF radio field strengths [Moler and Holden, 1960; Randall, 1962].

The effect of meteorological sensor time lag has been studied with the important conclusion that more accurate estimates of duct and elevated layer intensities may be obtained by careful reanalysis of the original sounding data [Wagner, 1960, 1961; Bean and Dutton, 1961]. In addition, the standard radiosonde instrument has been modified to yield more realistic measures of the refractive index profile [Clinger and Straiton, 1960; Deam, 1962].

Studies of meteorological data have indicated the possibility of extended ranges of VHF propagation within the tradewind inversion for the South Atlantic [Purves, Randall, and Ringwalt, 1959; Katzin et al., 1960]. Subsequent transmission loss measurements have confirmed these preliminary results. (See section 6, Guided Waves in the Troposphere.) Extension of this work to the elevated tradewind duct between San Diego and Hawaii indicates the same general conclusions as those for the South Atlantic but with the additional observation that the extended range of VHF signals was confined to a cloud-top layer of about 100-ft thickness [Randall, 1962]. These results have been confirmed and extended by the addition of radar studies of the azimuthal extent of the elevated ducting regions that indicate radar returns over a 1000 nautical miles extent of the California coastline to ranges as great as 900 nautical miles to sea compared to the normal range of 160 to 180 nautical miles [Hemenway and Randall, 1962].
A summary of a VHF radio meteorological experiment over the Pacific Ocean in 1946 has been prepared which essentially confirms the wartime Atlantic Ocean measurements off the island of Antigua, with the conclusion that no radio holes were observed during periods of extended ranges and that these extended ranges may be practically exploited for ship-to-ship and ship-to-shore communications for ranges up to 200 miles [Ament et al., 1962].

Experiments over a $104 \mathrm{~km}$ within-the-horizon over-water path in southern California on $394 \mathrm{Mc} / \mathrm{s}$ have shown a strong dependence of fading characteristics on the height of superrefractive layers above sea level. Prolonged spacewave fadeouts are much more likely to occur concurrent with ground-based and low elevated layers than with high layers or linear refractive index profiles. In these experiments, the terminals were located approximately 250 and $450 \mathrm{~m}$ above sea level, respectively [Barsis and Johnson, 1962].

The very strong changes of $N$ in the vicinity of cumulus clouds have been studied by Cunningham [1962]; a model of a cumulus cloud and its refractive index distribution has been developed with the important conclusion that radiosonde data may be used with sufficient accuracy to estimate the refractive index difference between the ambient air and the cloud interior. The occurrence of cumulus clouds geographically within the United States, diurnally and annually has been presented [Cunningham, 1962b].

The validity of the general exponential decrease of $N$ with height has been examined by Misme, Bean, and Thayer [1960] with the conclusion that this simple model is more widely applicable than at first supposed.

Comparative studies of the correlation of the diurnal and seasonal variation of monthly median VHF transmission losses in Germany and the United States. Bean, Fehlhaber, and Grosskopf [1962] have perhaps revealed the basis of much of the international debate as to the validity of the surface value of $N, N_{s}$, as a predictor of transmission loss variations. It is found that the variations of Western European transmission losses are characteristically much less than those from the eastern seaboard of the United States and, in addition, are frequently within or near the limit of measurement accuracy. The correlation of transmission loss with $N_{s}$ is found to be essentially the same on both continents when the range of transmission loss is large. Qualitative agreement with this conclusion is reached by climatic studies of $N_{s}$ which indicate a characteristic range for the Eastern United States of five times that of Western Europe.

Perhaps the most definitive conclusion in radio climatology reached during this reporting period is the experimental confirmation of $N_{s}$ as a predictor of atmospheric refraction for elevation angles in excess of a few degrees [Anway, 1963; Bean and Thayer, 


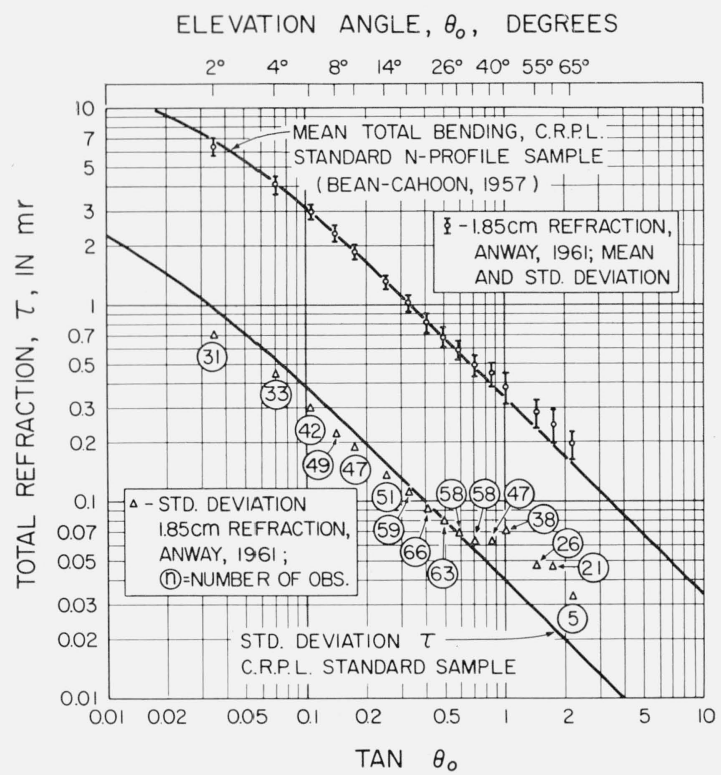

FiguRE 1.-Comparison of measured total atmospheric refraction of $1.85 \mathrm{~cm}$ radio waves at Cedar Rapids, Iowa, with values predicted from $\mathrm{N}_{\mathrm{s}}$.

Upper curve and data points: mean value of refraction; lower curve and data points: the standard deviation of the refraction values about the mean. The wings on the mean refraction data points indicate the limits of the standard deviation of the observed data.

1963]. These results, summarized in figure 1, show that the precision radio sextant measurements of total atmospheric refraction have been adequately predicted from surface meteorological data alone. Both the mean value of the total angular bending and the variation about this mean agree with previous estimates derived from meteorological data.

\section{References}

Ament, W. S., W. Binnian, W. W. Carter, M. Katzin, and D. T. Olmsted (Feb. 2, 1962), Microwave propagation measurements over the Pacific Ocean, 1946, NRL Report 5687.

Anway, A. D. (1963), Empirical determination of total atmospheric refraction at centimeter wavelengths by radiometric means. J. Res. NBS 67D (Radio Prop.), No. 2, $153-160$

Barsis, A. P., and M. E. Johnson (1962), Prolonged spacewave fadeouts in tropospheric propagation, J. Res. NBS $66 \mathrm{D}$ (Radio Prop.), No. 6, 681-699.

Bean, B. R., J. D. Horn, and A. M. Ozanich, Jr. Nov. 25, 1960), Climatic charts and data of the radio refractive index for the United States and the world, NBS Mono. 22, 178 pages.

Bean, B. R., and J. D. Horn (1961), On the average atmospheric radio refractive index structure over North America, Beitr. Phys. Atmo. 34, 1/2.
Bean, B. R., and E. J. Dutton (Nov. 1961), Concerning radiosondes, lag constants, and refractive index profiles, J. Geophys. Res. 66, No. 11, 3717-3723.

Bean, B. R., and G. D. Thayer (1963), Comparison of observed atmospheric radio refraction effects with values predicted through the use of surface weather observations, J. Res. NBS 68D (Radio Prop.), No. 3, 273-286.

Clinger, A. H., and A. W. Straiton (May 1960), Adaption of the radiosonde for direct measurement of radio refractive index, Bull. Am. Meteorol. Soc. 41, No. 5, 250-252.

Cunningham, R. M. (1962a), Cloud refractive index studies I, Geophys. Research Papers No. 51, AFCRC-TR-56-210, New Bedford, Mass.

Cunningham, R. M. (Jan. 1962b), Cumulus climatology and refractive index; cloud refractive index studies, II, Geophysical Research Papers No. 51, AFCRL, Bedford, Mass.

Deam, A. P. (Apr. 1962), Radiosonde for atmospheric refractive index measurements, Rev. Sci. Instr. 33, No. 4, 438441.

Gossard, E. E. (Mar. 1960), Power spectra of temperature, humidity and refractive index from aircraft and tethered balloon measurements, IRE Trans. Ant. Prop. AP-8, No. 2, 186-201.

Hemenway, D. F., and D. L. Randall (June 1962), Radarmeteorological observations of an elevated duct, Reprinted from NRL Progress Report.

Jehn, K. H. (June 1960a), Microwave refractive index distributions associated with the Texas-Gulf cyclone, Bull. Am. Meteorol. Soc. 41, No. 6, 304-312.

Jehn, K. H. (Feb. 1961), Microwave refractive-index distributions associated with the Central United States cold outbreak, Bull. Am. Meteorol. Soc. 42, No. 2, 71-76.

Jehn, K. H. (June 1960b), The use of potential refractive index in synoptic-scale radio meteorology, J. Meteorol. 17, No. 3, 264-269.

Katzin, M., H. Pezzner, B. Y. C. Koo, J. V. Larson, and J. C. Katzin (1960), The trade-wind inversion as a transoceanic duct, J. Res. NBS 64D (Radio Prop.), No. 3, $247-253$.

Misme, P., B. R. Bean, and G. D. Thayer (Aug. 1960), Models of the atmospheric radio refractive index, Proc. IRE 48, No. 8, 1498-1501.

Moler, W. F., and D. B. Holden (1960), Tropospheric scatter propagation and atmospheric circulations, J. Res. NBS, $64 D$ (Radio Prop.), No. 1, 81-93.

Purves, C. G., D. L. Randall, and D. L. Ringwalt (Sept. 1959; June 1960), Meteorological measurements in the South Atlantic, Reprint from NRL Progress Report; see also Meteorological measurements in the South Atlantic, Reprinted from NRL Progress Report.

Randall, D. L. (Apr. 1962), An application of potential vorticity theory to elevated radio-propagation ducts in stratus-type cloud layers, NRL Progress Report.

Randall, D. L. (May 1962; Apr. 1961), Project tradewinds IV: Radiometeorological conditions in the elevated duct between San Diego and Honolulu (June 22 and 26, 1961), Reprint from NRL Progress Report. See also the elevated duct between San Diego and Hawaii, Reprint from NRL Progress Report.

Wagner, N. K. (May 1961), The effect of time constant of radiosonde sensors on the measurement of temperature and humidity discontinuities in the atmosphere, Bull. Am. Meteorol. Soc. 42, No. 5, 317-320.

Wagner, N. K. (July 1960), An analysis of radiosonde effects on the measured frequency occurrence of ducting layers, J. Geophys. Res. 65, No. 7, 2077-2085. 


\section{Radar Meteorology and Cloud Physics}

\subsection{Severe Local Storms}

Detection, identification, and tracking of severe thunderstorms, tornadoes, and hailstorms have probably given radar meteorology its greatest boost in the United States. Photographic records of the plan position indicator (PPI) display of three tornadoes in 1953 led to the establishment of a network of radars by the U.S. Weather Bureau in cooperation with city and local governments. Subjective scope interpretation during the mid-1950's proved to be reasonably successful and is credited with saving many lives. A need for objective identification methods led to the use of quantitative measurements of echo intensity and height. Investigations by Donaldson [1961], Wilk [1961], and Austin [1961] showed that at 3-cm wavelength, a high radar reflectivity and/or echo tops reaching or exceeding the tropopause were excellent indicators of tornadoes or hail at the surface. However, validity of radar echo heights has been challenged recently by Jordan [1962], who reported a wide variance in echo heights reported by radars in close proximity. In a study of simultaneous echo top measurements and visual cloud top measurements, Saunders and Ronne [1962] found angular differences of 0 to $+1^{\circ}$ with the radar elevation higher. Important to a program of absolute echo intensity measurements is proper calibration of the radar equipment. Atlas and Mossop [1960] have suggested a method utilizing small balloon-borne spheres.

A major effort directed toward a better understanding of severe storm mechanisms has been initiated by the U.S. Weather Bureau. A number of instrumented aircraft fly carefully controlled and monitored tracks around and through squall lines and thunderstorms while several ground-based weather radars monitor the three-dimensional distribution of echo areas and intensities, and special surface network and upper-air sounding stations record temperature, wind humidity, pressure, and rainfall. A detailed analysis of a radar echo associated with a tornado has recently been completed [Donaldson, 1962].

\subsection{Hurricanes}

As a result of several disastrous hurricanes in 1955 , a network of weather radars was installed along the Atlantic and Gulf coasts of the United States during 1960 [Bigler, Hexter, and Wells, 1962]. These equipments were used very successfully on several occasions, providing hurricane forecasters with far better hurricane eye position information than ever before. Data from these radars as well as others were used by Senn [1961] for detailed studies of eve motion and the structure of hurricanes [Jordan, Hurt, and Lowrey, 1960; Jordon, Schatzle, and Cronise, 1961; Conover, 1961].

\subsection{Forecast and Synoptic Applications}

By their nature, radar observations indicate the time, space, and intensity variations of precipitation. Since many weather forecast problems are concerned with predictions of parameters affected by precipitation, radar has an important input to this aspect of meteorology. Progress, however, has not been as rapid as it could be, owing to difficulties associated with the relay of information from the radar console to the forecaster in a remote location. An appraisal of the use of radar in forecasting has been prepared by Kessler [1961]. A study of some of the statistical properties of radar echoes has been completed by Hilst and Russo [1960]. These, coupled with studies by Boucher [1961] on the motion and prediction of precipitation lines and areas, point the way toward improved techniques for the use of radar. Usefulness of coded radar reports and summary facsimile charts in short-period forecasting has been demonstrated [Wilson and Kessler, 1962], and the value of composited radarscope photographs in the U.S. man-in-space program has been illustrated [Hoose, 1962].

Radar echoes associated with mountain wave clouds have been observed and described by Colson, Lindsay, and Hand [1961]. The observations show agreement with theory on the wavelength of mountain waves under the conditions of atmospheric stability that existed at the time of the observations.

\subsection{Hydrology}

Observation of heavy and persistent rainfall by radar is very useful to the hydrologist responsible for flood forecasting. For these purposes, radars of 5 - or $10-\mathrm{cm}$ wavelength are required to minimize effects of attenuation. As stated by Hamilton and Marshall [1961], truly quantitative operation, i. e., measurement of rainfall rate, "demands" use of $10-\mathrm{cm}$ wavelength. However, Collis [1962] has reported on the feasibility of using $X$ - or $K$-band radars for rainfall measurements over relatively small areas by measurement of the amount of signal attenuation. Methods for applying radar information to hydrology have been described by Flanders [1961], Leiber, Merritt, and Robertson [1961], and McCallister [1961]. Radar measurements of areal rainfall reported by Kodaira [1961] indicate that accuracy of the order of \pm 50 percent may be achieved.

\subsection{Weather Radar and Satellites}

Availability of a large quantity of TIROS cloud photographs has inevitably led to studies of the relationship between radar echo areas and cloud patterns which have been reported by Nagle and Serebreny [1962], Blackmer [1961], Fujita et al. [1962], and Fujita [1961]. Although rectification of cloud photographs forces painfully slow progress 
in this area of endeavor, results thus far indicate that the two observations complement each other very well.

\subsection{Non-Precipitation Echoes (Angels)}

Controversy over the cause of these extremely interesting echoes continues. In some cases, echoes are clearly the result of birds [Ligda, 1958]; however, many scientists in the United States favor refractive index anomalies as the cause in most instances. Atmospheric refractive index models have been developed by Harney [1961] and Atlas [1960a] to explain "angels." Detection of a sea-breeze front by $K$-band radars has been described by Atlas [1960b], and illustrations of $S$-band detection have been presented by Bigler, Hexter, and Wells [1962]. Additionally, some of the characteristics of "angel" echoes have been described by Vrana [1961]. Roelofs and Bolgiano [1962] have reported tracking clear-air targets. Individual "angels" were detectable for periods in excess of $20 \mathrm{~min}$; the velocities of the targets deviated considerably from the radiosonde winds and the radar cross sections were a sensitive function of elevation angles.

\subsection{Measurement of Air Motion by Radar}

Emphasis is being placed on obtaining an indication of air motion in the atmosphere by use of radar. For the most part, the measurement involves a study of the velocities of hydrometeors suspended or falling through the air. Some effort also is being made to obtain an indication of clear air turbulence; in this case the radar return is from local refractive index gradients. L'hermitte and Atlas [1961] have developed a method for measuring wind and particle fall velocity by means of a pulse Doppler radar. By using a beam tilted up from the horizon and by scanning $360 \mathrm{deg}$ in azimuth, one can obtain wind and particle fall velocity under uniform precipitation conditions. Data collected with the $C$-band radar compare favorably with other wind measurements [Atlas, 1962; L'hermitte, 1962].

Probert-Jones and Harper [1961] have mapped vertical velocities in showers from data supplied by a Doppler radar with its antenna pointing vertically upward, while Rogers and Pilie [1962] have made measurement of the drop size distribution in rain using Doppler radar techniques.

The U.S. Weather Bureau has used a WSR-57 10-cm radar at Evansville, Ind., to investigate the feasibility of detecting clear-air turbulence [Stewart, 1961]. Observations of clear-air turbulence by aircraft pilots within range of the radar are compared with indications of turbulence on the radar. A study of Stackpole [1961] on the response of raindrops to turbulent flow indicates there may not be a strong relationship between the radar indication of turbulence and the actual air turbulence.

\subsection{Attenuation and Scattering}

Theoretical and experimental work is being carried on to improve our knowledge of scattering by water and ice. Discrepancies still exist between the radar return predicted from rainfall measurements and that actually measured. In a paper by Probert-Jones [1961], a detailed rederivation of the radar equation suggests that the theory applied to a meteorological target at present is incorrect; a modification of the equation brings theory and experiment into good agreement. Meanwhile, more effort is being expended to delve into the physics of the scattering problem, both analytically and experimentally with emphasis on nonspherical shapes.

Herman and Battan [1961] have made new computations of attenuation and scattering of an electromagnetic wave incident on ice spheres. Their results show that as the ice particle increases in size, the forward scattering also increases. Since this forward-scattered energy adds to the incident energy, a correction to the attenuation must be made; this correction applies to calculations of attenuation by hailstones with diameters in excess of $1.5 \mathrm{~cm}$. Values of attenuation have been carried out for diameter-to-wavelength ratios up to three. In addition, they have calculated the backscattering, attenuation, absorption, and scattering cross sections for ice spheres consisting of mixtures of ice and water [Battan and Herman, 1961]. A study of the polarization properties of nonspherical hail indicates that the greatest reflectivity on a $3-\mathrm{cm}$ radar will be seen with vertical polarization. On a $10-\mathrm{cm}$ radar, however, these reflectors will present a cross section as much as $10 \mathrm{db}$ below that of an equivalent ice sphere for either polarization, but with horizontal slightly higher than vertical.

This same subject was studied experimentally by Atlas and Wexler [1961]. Measurements of backscatter from nonspherical ice spheroids, 2.8 to 5.1 $\mathrm{cm}$ in diameter, were made in the laboratory at radar wavelengths of 9.7 and $3.2 \mathrm{~cm}$. At $9.7 \mathrm{~cm}$ the backscatter is well-behaved, i.e., the largest return occurs when the radar views the largest face of the spheroid. At $3.2 \mathrm{~cm}$ the backscatter patterns are more complicated, showing several maxima as the spheroid is rotated. The results at $9.7 \mathrm{~cm}$ show similarities to Rayleigh scattering, while at $3.2 \mathrm{~cm}$ the irregularities in pattern are consistent with the large fluctuations of cross sections in the middle of the Mie region.

Other measurements of radar cross sections of water drops and ice spheres were made at the EERL of the University of 'Texas [Gerhardt, Tolbert, Brunstein, and Bahn, 1961; Gerhardt, Tolbert, and Brunstein, 1961; Stephens, 1961]. In this work, wavelengths of $1.5,3.2$, and $5.7 \mathrm{~cm}$ were used. The range of sizes of water drops was from 0.2 to $0.7 \mathrm{~cm}$, for ice 0.3 to $3.5 \mathrm{~cm}$. For $\lambda=3.2 \mathrm{~cm}$, scattering from water drops up to $0.35 \mathrm{~cm}$ was found larger than the Mie and Rayleigh scattering and agreed with Mie theory for larger sizes. Cross sections for ice agreed well with the theoretical values of Mie. For $\lambda=5.7$ $\mathrm{cm}$ Rayleigh values are applicable up to drop diameters of $0.35 \mathrm{~cm}$; for $\lambda=1.5 \mathrm{~cm}$ Rayleigh values are applicable up to $0.15 \mathrm{~cm}$. For ice spheres with a water film thickness of $0.01 \mathrm{~cm}$, this experimental work and the theoretical work of Herman and Battan [1961] were in complete agreement. 
In an effort to study the return from the smaller droplets in clouds, a droplet collector was developed for use on an airplane ['Todd, 1961]. Droplets are collected on clear polyester motion-picture film coated with Formvar; the coated film is softened with ethylene dichloride just prior to exposure to the cloud. Cloud particles embed themselves in the softened plastic. When the film is dried it contains a permanent record of samples of the water and ice crystal distributions in the cloud. These distributions are compared with the radar return measured on a ground-based $X$-band radar.

\section{References}

Atlas, D., and S. C. Mossop (July 1960), Calibration of a weather radar using a standard target, Bull. Am. Meteorol. Soc. 41, 7 .

Atlas, D. (Apr. 1960a), Possible key to the dilemma of meteorological "Angel" echoes, J. Meteorol. 17, 2.

Atlas, D. (June 1960b), Radar detection of the sea breeze, J. Meteorol. 1\%.

Atlas, D. (Sept. 1962), Indirect probing techniques, Bull. Am. Meteorol. Soc. 43, 9 .

Atlas, D., and R. Wexler (Oct. 1961), Radar scatter by large nonspherical hail, Pro. Ninth Weather Radar Conf.

Austin, P. M. (Aug. 1961), Research directed toward the investigation of radar techniques for severe storm identification and the measurement of precipitation growth, Massachusetts Institute of Technology, Department of Meteorology, Final Report under Contract AF 19(604)2291.

Battan, L. J., and B. M. Herman (Oct. 1961), The radar cross-sections of "spongy" ice spheres, Proc. Ninth Weather Radar Conf.

Bigler, S. G., P. L. Hexter, Jr., and F. E. Wells (Nov. 1962), Radar program of the U.S. Weather Bureau, Bull. Am. Meteorol. Soc. 43, 11.

Blackmer, R. H. (Oct. 1961), Satellite observations of squall line thunderstorms, Proc. Ninth Weather Radar Conf.

Boucher, R. J. (June 1961), Weather radar research and applied techniques, Allied Research Associates, Final Report Under Contract AF 19(604)-5204.

Collis, R. (June 1962), Study of techniques for measuring rainfall by reference to radar attentuation, Stanford Research Institute Project 3948, Final Report under Contract CWB 10271

Colson, D. V., C. V. Lindsay, and J. N. Hand (Jan. 1961), Radar echo of a mountain wave on February 15, 1960, Monthly Weather Rev. 89, 1.

Conover, L. F. (June 1961), Evaluation of eye fixes obtained by radar for Hurricane Donna, U.S. Weather Bureau National Hurricane Research Project Report No. 50, Proceedings of the Second Technical Conference on Hurricanes.

Donaldson, R. J. (June 1961), Radar reflectivity profiles in thunderstorms, J. Meteorol. 18, 3.

Donaldson, R. J. (Apr. 1962), Radar observations of a tornado in vertical section, Report No. 8, U.S. Weather Bureau Nation Severe Storms Project.

Flanders, A. F. (Oct. 1961), The Weather Bureau's radarhydrology program, Proc. Ninth Weather Radar Conf.

Fujita, T., T. Ushijima, W. A. Hass, and G. T. Dellert (Apr. 1962), Meteorological interpretation of convective nephsystems appearing in TIROS cloud photographs, Univ. of Chicago Mesometeorology Project Research Paper No. 9 under Contract CWB 10215.

Fujita, T. (June 1961), A method of combining radar, aerial, and satellite photographs taken during hurricanes, U.S. Weather Bureau National Hurricane Research Project No. 50, Proc. Second Tech. Conf. on Hurricanes.

Gerhardt, J. R., C. W. Tolbert, S. A. Brunstein and W. W. Bahn (June 1961), Experimental determinations of the backscattering cross-sections of water drops and of wet and dry ice spheres at 3.2 centimeters, J. Meteorol. 18, 3 .

Gerhardt, J. R., C. W. Tolbert, and S. A. Brunstein (Oct. 1961), Further studies of the back-scattering cross-sec- tions of water drops and wet and dry ice spheres, J. Meteorol. 18,5 .

Hamilton, P. H., and J. S. Marshall (Jan. 1961), Weatherradar attenuation estimates from rain gauge statistics, MeGill University Scientific Report MW-32, under Contract AF 19 (604)-2065.

Harney, P. J. (Oct. 1961), A new explanation for anomalous radar observations, Proc. Ninth Weather Radar Conf.

Herman, B. M., and L. J. Battan (Oct. 1961), Calculations of the total attenuation and angular scatter of ice spheres, Proc. Ninth Weather Radar Conf.

Hilst, G. E., and J. A. Russo (Nov. 1960), An objective extrapolation technique for semi-conservative fields with an application to radar patterns, Travelers Research Center Technical Memorandum No. 3 under Contract AF 30 (635) -14459 .

Hoose, H. M. (Sept. 1962), Operational use of weather radar composites in Florida, Monthly Weather Rev. 90, 9.

Jordan, C. L. (Apr. 1962), On the maximum vertical extent of convective clouds, Department of Meteorology, Florida State University, Scientific Report under Contract N189$50775 \mathrm{~A}$

Jordan, C. L., D. A. Hurt, and C. A. Lowrey (June 1960), On the structure of Hurricane Daisy on 27 August 1958, J. Meteorol. 1\%, 3.

Jordan, C. L., F. S. Schatzle, and W. C. Cronise (Oct. 1961), Some interesting radar features of Hurricane Donna, Proc. Ninth Weather Radar Conference.

Kessler, E. (Oct. 1961), An appraisal of the use of radar in observation and forecasting, Proc. Ninth Weather Radar Conf.

Kodaira, N. (Oct. 1961), Radar areal-rainfall measurements, Proc. Ninth Weather Radar Conf.

Leiber, G., C. J. Merritt, and J. P. Robertson (Oct. 1961), WSR-57 Analysis of heavy rains, Proc. Ninth Weather Radar Conf.

L'hermitte, R. and D. Atlas (Oct. 1961), Precipitation motion by pulse Doppler, Proc. Ninth Weather Radar Conf.

L'hermitte, R. (July 1962), Note on wind variability with Doppler radar, J. Atmospheric Sci. 19, 4.

Ligda, M. G. H. (Dec. 1958), Radar observations of blackbird flights, Texas J. Sci.

McCallister, J. P. (Oct. 1961), Radar observations in hydrologic analysis, Proc. Ninth Weather Radar Conf.

Nagle, R. E., and S. M. Serebreny (Sept. 1962), Radar precipitation echo and satellite cloud observations of a maritime cyclone, J. Appl. Meteorol. 1, 3.

Probert-Jones, J. R., and W. G. Harper (Oct. 1961), Vertical air motion in showers as revealed by Doppler radar, Proc. Ninth Weather Radar Conf.

Probert-Jones, J. R. (Oct. 1961), The radar equation in meteorologv, Proc. Ninth Weather Radar Conf.

Roelofs, T. H., and R. Bolgiano, Jr. (Apr. 1962), A preliminary study of radar angels at Wallops Island, Virginia, a paper presented at URSI Meeting.

Rogers, R. R., and R. J. Pilie (Nov. 1962), Radar measurements of drop size distribution, J. Atmospheric Sci. 19, 6 .

Saunders, P. M., and F. C. Ronne (Sept. 1962), A comparison between the height of cumulus clouds and the height of radar echoes received from them, J. Appl. Meteorol. 1, 3.

Senn, H. V. (Oct. 1961), Hurricane eye motion as seen by radar, Proc. Ninth Weather Radar Conf.

Stackpole, J. D. (Oct. 1961), The effectiveness of raindrops as turbulence sensors, Proc. Ninth Weather Radar Conf.

Stephens, J. J. (June 1961), Radar cross-sections for water and ice spheres, J. Meteorol. 18, 3.

Stewart, P. G. (Oct. 1961), Radar in turbulence research, Proc. Ninth Weather Radar Conf.

Todd, Clement J. (Oct. 1961), A study of cloud composition, Proc. Ninth Weather Radar Conf.

Vrana, N. (Sept. 1961), Some characteristics of radar angel echoes, Cornell University Research Report RS 32 under Contract AF 19(604)-6160.

Wilk, K. E. (Dec. 1961), Research concerning analysis of severe thunderstorms, Illinois State Water Survey, Final Report under Contract AF 19(604)-4940.

Wilson, J. W. and E. Kessler (July 1962), Use of radar summary maps for weather analysis and forecasting, Travelers Research Center Technical Report No. 14 under FAA Contract BRD-363. 


\section{Tropospheric Propagation Affecting Space Communications}

There are three categories into which tropospheric propagation effects important to space communications can be subdivided, namely: refractive effects, these being of importance in tracking communication satellites at low angles of elevation with very narrowbeam antennas; absorptive effects, of importance in both the clear and the precipitant atmosphere because of the attenuation and the noise which they introduce; scattering effects, due to both inhomogeneity in refractive index and precipitation in the atmosphere, these being media which introduce both interference and thermal noise into the space communication system.

\subsection{Refractive Effects}

Methods of calculating tropospheric refraction, knowing the magnitude of the refractive index at the earth's surface, have been developed [Bean and Thayer, 1959], and these have been shown to agree well with experimental data obtained using the radio sextant [Iliff and Holt, 1963; Anway, 1963]. Tracking of satellites with narrow-beam antennas may therefore be accomplished using methods which rely on predicted orbital elements, provided corrections that take refractive index profile characteristics into account are made for low angles of elevation. Deviation in the angle of arrival of the radio waves from the average and predicted values has also been studied [Bean, Horn, and Riggs, 1960; Bean and Dutton, 1960; Bean, 1961; Anderson, Beyers, and Rainey, 1960].

\subsection{Absorptive Effects \\ a. Clear Atmosphere-Attenuation}

The absorption by oxygen and water vapor results in significant attenuations at millimeter wavelengths (a summary is given by Straiton and Tolbert [1960]) therefore, even under clear atmospheric conditions, these wavelengths are not especially attractive for space communications (see, however, Straiton and Tolbert [1963]). Measurements of ground-level attenuation have been extended to frequencies above $100 \mathrm{Gc} / \mathrm{s}$ [Tolbert and Straiton, 1959]; and it is found, as with investigations at somewhat lower frequencies, that the loss due to oxygen agrees well with theoretical prediction, whereas that due to water vapor is significantly larger than expected; no suitable explanation has yet been given for the discrepancy.

Calculations of the attenuation of centimeter waves through the entire troposphere for an antenna beamed at various angles of elevation have been made by Blake [1961] and Hogg [1959]. It is found that the attenuation per se is not significant for wavelengths greater than $3 \mathrm{~cm}$ provided the elevation of the antenna beam exceeds $5 \mathrm{deg}$.

\section{b. Clear Atmosphere-Sky Noise}

Low-noise amplifiers and antennas are a necessity in space communications because of the large distances involved and the need to conserve power in the satellites [Pierce and Kompfner, 1959]. With present technology, the limiting factor in the sensitivity of a centimeter-wave receiving system is the sky noise which is generated by the absorption discussed in section a. Calculation [Hogg, 1959; Blake, 1961; Bean, Abbott, and Westwater, 1962] and experiment [DeGrasse et al., 1959; Ohm, 1961; Hogg and Semplak, 1961a] show that the effective sky noise temperature due to oxygen is about $3^{\circ} \mathrm{K}$ for zenith orientation and about $100{ }^{\circ} \mathrm{K}$ for horizon orientation of the antenna beam at the longer centimeter wavelengths. The noise produced by water vapor increases these values somewhat; the increase is a function of frequency and also of season because of the changing water vapor content of the atmosphere at a given latitude. Losses in the atmosphere also enter into calculation of the effect of environment on the noise temperature of antennas [Chen and Peake, 1961; Hogg, 1961]. Ring [1962] discusses extensive measurements at $35 \mathrm{Gc} / \mathrm{s}$ of sky noise and the effect of environment on antenna temperature. The noise generated by the troposphere in an antenna on a satellite has been estimated [Weger, 1960].

\section{Precipitates-Absorption and Sky Noise}

Attenuation by rain at millimeter wavelengths essentially prohibits their use for reliable earth-space communication; however, the effect decreases rapidly toward the long wavelength end of the microwave band. No measurements of attenuation by rain of signals from a source beyond the troposphere have yet been published in the United States.

The sky noise generated by rain is readily observable; effective noise temperatures at $6 \mathrm{Gc} / \mathrm{s}$ in excess of $110^{\circ} \mathrm{K}$ due to rain have been observed [Hogg and Semplak, 1961a,b]. As in the case of absorption, the total emission of sky noise depends on the rate of rainfall and the length of the propagation path through the rain. Estimates of sky noise due to rain for the entire centimeter-wave band have been made [Hogg and Semplak, 1963].

Since snow is a relatively lossless medium at centimeter wavelengths, it produces negligible attenuation and sky noise. 


\subsection{Scattering Effects}

\section{a. Precipitates}

Thermal noise can also be introduced into a highly sensitive receiving system by the scattering process. In this case the sources of the thermal noise are the surrounding atmosphere (which has a relatively cool effective temperature) and the earth (which, if perfectly absorbing, has an effective temperature of about $300{ }^{\circ} \mathrm{K}$ ); the radiation from both of these sources propagates into the rain where it is scattered toward the receiving antenna. The noise produced by scattering is believed to be small relative to that produced by emission.

Scatter by rain of radiation from interfering transmitters located some distance from the receiving site, but operating in the same frequency band, can degrade the performance of a space communication system. This effect has been discussed on a theoretical basis by Dennis [1961]. In an experiment [Hogg, Semplak, and Gray, 1963] at $4 \mathrm{Gc} / \mathrm{s}$ during a heavy thunderstorm, a radio relay transmitter located 20 miles from a vertically beamed receiving antenna produced interference six times the thermal noise caused by the rain. This effect is also discussed by Doherty and Stone [1960].

\section{b. Tropospheric Scatter Propagation}

In a highly sensitive receiver, significant interference can also be produced via trophospheric scatter propagation from transmitters beyond the horizon, especially under abnormal conditions of strong layers or of ducting. This interference presents a problem in frequency allocation and siting if frequency bands are to be shared by radio relay and space communication systems. In evaluating the effect, the "common volume" concept so often used in scatter propagation is not generally applicable since it is more often than not a question of the main beam of the one antenna scattering into the wide-angle side lobes of the other.

\section{References}

Anderson, W. L., N. J. Beyers, and R. J. Rainey (1960), Comparison of experimental with computed tropospheric refraction, IRE Trans. Ant. Prop. AP-8, No. 5, 456.

Anway, A. O. (1963), Empirical determination of total atmospheric refraction at centimeter wavelengths by radiometric means, J. Res. NBS.
Bean, B. R., and G. D. Thayer (1959), Models of the atmospheric radio refractive index, Proc. IRE 4\%, No. 5, 740.

Bean, B. R., J. D. Horn, and L. P. Riggs (1960), Refraction of radio waves at low angles within various air masses, J. Geophys. Res. 65, No. 4, 1183.

Bean, B. R., and E. J. Dutton (1960), On the calculation of the departures of radio wave bending from normal, J. Res. NBS 64D, No. 3, 259.

Bean, B. R. (1961), Comparison of observed tropospheric refraction with values computed from the surface refractivity, IRE Trans. Ant. Prop. AP-9, 4.

Bean, B. R., R. L. Abbott, and E. R. Westwater (1962), Analysis of thermal noise temperature as a remote probe of atmospheric structure, NBS Report No. 7293.

Blake, L. V. (1961), Antenna and receiving system noise calculation, U.S. Naval Res. Lab. Rept. No. 5668.

Chen, S. N. C., and W. H. Peake (1961), Apparent temperature of smooth and rough terrain, IRE Trans. Ant. Prop. AP-9, No. 6, 567 .

DeGrasse, R. W., D. C. Hogg, E. A. Ohm, and H. E. D. Scovil (1959), Ultra-low-noise receiving system for a satellite or space communication, Proc. Natl. Electronic Conf. 15, 370.

Dennis, A. S. (1961), Forward scatter from precipitation as an interference source at stations monitoring satellites, Stanford Res. Memo. No. 2 on Project 3773.

Doherty, L. H., and S. A. Stone (1960), Forward scatter by rain, IRE Trans. Ant. Prop. AP-8, No. 4, 414.

Hogg, D. C. (1959), Effective antenna temperatures due to oxygen and water vapor in the atmosphere, J. Appl. Phys. 30, 1417 .

Hogg, D. C. (1961), A study of the sources of noise in centimeter wave antennas, Low Noise Electronics (Pergamon Press, New York, N.Y.), p. 307.

Hogg, D. C., and R. A. Semplak (1961a), Effect of rain and water vapor on sky noise at centimeter wavelengths, Bell System Tech. J. 40, 1331.

Hogg, D. C., and R. A. Semplak (1961b), Effect of rain on the noise level of a microwave receiving system, Proc. IRE 48, 12 .

Hogg, D. C., and R. A. Semplak (Mar. 1963), Estimated sky temperatures due to rain for the microwave band, Proc. IEEE 51, No. 3, 499.

Hogg, D. C., R. A. Semplak, and D. A. Gray (Mar. 1963), Measurement of microwave interference at $4 \mathrm{Gc}$ due to scatter by rain, Proc. IEEE 51, No. 3, 500.

Iliff, W. R., and J. M. Holt (1963), Use of surface refractivity in the empirical prediction of total atmospheric refraction, J. Res. NBS.

Ohm, E. A. (1961), Receiving system, Project Echo, Bell Syst. Tech. J. 40, No. 4, 1065.

Pierce, J. R., and R. Kompfner (1959), Transoceanic communications by means of satellites, Proc. IRE 4\%, 372 .

Ring, D. H. (1962), Final report AF-19-(122)-458 to Lincoln Laboratories, 1956.

Straiton, A. W., and C. W. Tolbert (1960), Anomalies on the absorption of radio waves by atmospheric gases, Proc. IRE 48, No. 5, 898 .

Straiton, A. W., and C. W. Tolbert (1963), Factors affecting earth-satellite millimeter wavelength communications, IRE Trans. Microwave Theory Tech. MTT.

Tolbert, C. W., and A. W. Straiton (1959), Radio propagation measurements in the 100 to 118 Kmes spectrum, IRE Wescon Conv. Record, Part 1, 56.

Weger, E. (1960), Apparent thermal noise temperatures in the microwave region, IRE Trans. Ant. Prop. AP-8, 2. 


\section{Influence of Irregularities of Terrain and of Vegetation on Radio Wave Propagation}

Irregularities and vegetation on the earth's surface contribute in a great many ways to the propagation of radio waves through, around, to, or from the surface of the earth. Information pertaining to terrain and vegetation effects is related to several of the Commissions of URSI and is in part incorporated in the reports of these other commissions and in other sections of the Commission 2 report. For example, the propagation of radio waves through the troposphere is definitely influenced by the surface effects, and such problems as the characteristics of propagation over mixed land and sea surfaces have been described in the Commission 4 report. Backscattering, as related to arbitrary geometrical surfaces, has also been described in Commission 4 reports but to the extent that these surfaces serve as models of the ground, they overlap the interests of Commission 2. These subjects will, therefore, not be included in the Commission 2 report.

Five basic areas will be described in this section as follows:

1. The effect of terrain and foliage features on the signal strength as measured and as calculated at various locations on irregular terrain.

2. Backscatter and forwardscatter of rough surfaces on the earth, particularly at near grazing incidence.

3. Reflection from the earth at near vertical incidence.

4. The effect of foliage and earth features on the radiation from the earth as seen by a radiometer above the earth's surface.

5. The effect of the earth's surface in controlling the turbulence characteristics of the troposphere itself.

\subsection{Effects of Terrain and Vegetation on Signal Characteristics}

Considerable work was done by the Television Allocations Study Organization (TASO) to develop more reliable VHF and UHF propagation curves for use in allocation work by the Federal Communications Commission. LaGrone [1960] analyzed considerable VHF and UHF field data taken under field conditions varying from smooth plains to rugged mountainous terrain and developed empirical equations from which the effects of terrain on the signal could be computed. He found the average absorption by vegetation to be approximately $1 \mathrm{db}$ at low VHF, $4 \mathrm{db}$ at high VHF, and $22 \mathrm{db}$ at low UHF. LaGrone also developed new propagation curves for high and low VHF and low UHF. Head [1960] was concerned with the effects of dense vegetation (trees) in the foreground of the receiving antenna on the received signal amplitude. Measurements were reported at $485 \mathrm{Mc} / \mathrm{s}$. He found the UHF signal to be approximately $30 \mathrm{db}$ below the smooth earth value close behind the trees and $10 \mathrm{db}$ below at a distance of about one mile from the trees. The receiving antenna was $30 \mathrm{ft}$ above local ground and the trees had an average height of $60 \mathrm{ft}$. $\mathrm{He}$ also found the average effect of groves of varying densities to be $-22 \mathrm{db}$. This agrees with the results reported by LaGrone [1960].

Further studies at The University of Texas of the effects of vegetation on the received signal were reported by LaGrone and Chapman [1961] and by LaGrone, Martin, and Chapman [1963]. LaGrone and Chapman made measurements at very low angles of $2,880 \mathrm{Mc} / \mathrm{s}$ signals over wooded areas as a function of the elevation angle of the transmitter. The effects of one tree and of a grove of trees on the apparent location of a signal source as determined by a narrow beam antenna were evaluated and reported. LaGrone, Martin, and Chapman made height gain signal strength measurements at various distances behind a dense grove of trees in full leaf. The measurements were made at $82,210,633,1280$, and $2950 \mathrm{Mc} / \mathrm{s}$. Theoretical diffraction curves for a smooth spherical earth and for a knife edge were compared with the measured height gain curves. Excellent agreement between the measured signal and the knife-edge diffracted signal was found at the higher frequencies, with significant agreement in evidence at the lower frequencies.

Barsis and Kirby [1961] have reported measurements of long-term signal variations at 100 and 750 $\mathrm{Mc} / \mathrm{s}$ over a long $(223 \mathrm{~km})$ knife-edge diffraction path. While the agreement between the observed transmission loss and that predicted using standard diffraction theory was not particularly good even when allowance is made for rounding of the knife edge [Wait and Conda, 1959], they found the longterm variations in hourly medians tended to behave like two line-of-sight paths in tandem. Short-term variations resembled space-wave fadeouts earlier defined for within-the-horizon paths; periods of signal enhancements were also observed and analyzed. It would appear that the shielding effect of large mountains is questionable, particularly if knife-edge type propagation paths are involved. Furutsu [1963] has derived rigorous solutions to propagation over terrain models with multiple diffracting obstacles such as cones, cylinders, and bases; the predicted results agree very well with laboratory measurements made at $23,000 \mathrm{Mc} / \mathrm{s}$ on a double knife-edge setup.

\subsection{Scattering at Angles Near Grazing Incidence}

\section{a. Backscatter}

Laboratory measurements have been made on water surfaces by Schooley [1962] to obtain statis- 
tical distributions of facet size which depend on wind speed, facet slope, and flatness tolerance. He defines flatness tolerance to be 10 percent of the radiofrequency wavelength. Using laboratory data, he calculates the upwind-to-downwind ratio of radar backscatter as a function of depression angle for various wind speeds and radar wavelengths and compares them with experimental results.

The Naval Research Laboratory has conducted flights with their radar-equipped airplane to obtain measurements of radar cross sections of ships and the sea surface. Ransone, Davis, and Daley [1962] report reflectivity values of a radar picket ship and a tanker for various ship aspect angles and radar depression angles. Radar cross sections are given for a sea with 3-ft waves. The transmitter in these experiments was vertically polarized; on reception, both vertical and horizontal polarization were used. It was found that at $90 \mathrm{deg}$, for example, the contrast between the ship and the sea background improved by 10 to $15 \mathrm{db}$ when receiving on horizontal polarization.

An experiment was performed to obtain reflectivity of the ocean, ice, and land at high frequencies-32.8 Mc/s. Nielsen, Hagn, Rorden, and Clark [1960], Stanford Research Institute, reported the results of this investigation. They conclude that the electrical properties of terrain lose their importance at small depression angles, while both roughness and surface electrical properties become important at high angles. Return from ice depends more on the physical characteristics of the surface than on the electrical properties. Water, smooth ice, and mixed land and water surfaces give higher return on vertical polarization than on horizontal, while land surfaces give equal return on both polarizations.

Reports that radar altimeters were in error over thick ice layers have been prevalent since about 1946. More recent studies have shed light on dielectric constants, scattering, and interface reflections of continental ice. Waite and Schmidt [1962] discuss recent work at frequencies of 110,220 , and 440 $\mathrm{Mc} / \mathrm{s}$ which indicates that radar altimeters operating at any of these frequencies can give fatally dangerous height indications when used by low-flying pilots over thick ice. On the other hand, frequency modulation altimeters operating at $4300 \mathrm{Mc} / \mathrm{s}$ provide accurate altitude measurements.

\section{b. Forward Scatter}

Experimental studies of over-water propagation on line-of-sight paths in the Gulf of Mexico are reported by Beard [1961]. The results are compared with theoretical and experimental work published earlier. The coherent field is somewhat higher than that theoretically predicted; the incoherent scattered power, if plotted against ocean roughness, rises to a maximum then exhibits a downtrend with increasing roughness. Probing of the reflected energy with a narrow-beam antenna shows that the coherent and incoherent power come mainly from the specular direction. The results indicate that multiple scattering theory may be needed for an adequate explanation of the data. Katz [1962] has extended the phenomenological model of forward scattering to include circular polarization. He derives the values for reflection coefficients for the rough-sea case and gives analytical expressions for the total received signal. He includes the coherent and incoherent components for receiver polarizations of the same and opposite senses to that of the transmitter.

\subsection{Reflection from the Earth at Near Ver- tical Incidence}

Radar terrain return studies at the University of New Mexico have been concerned with the theoretical interpretation of a large amount of data taken by Sandia Corporation using near vertical incidence radar soundings and acoustical models of these soundings. Edison, Moore, and Warner [1960] reported a theoretical study of the Sandia data at $450 \mathrm{Mc} / \mathrm{s}$. The radar cross section per unit area at vertical incidence ranged between values of 0.7 for wooded areas to approximately 4 for city targets, while at $3800 \mathrm{Mc} / \mathrm{s}$ the variation ranged from 0.8 for woods to about 18 for city targets. The scattering cross section for most targets drops off rapidly as the angle of incidence is increased. An exception is wooded areas which are nearly isotropic targets. The range of fading varied from about $3 \mathrm{db}$ for smooth water surfaces exhibiting principally specularly returns to about $19 \mathrm{db}$ for certain nonhomogeneous target areas.

As a supplemental study to the radar return, an acoustics simulator for modeling backscattering was developed and was reported by Edison [1961] and by Edison, Moore, and Warner [1961]. It was determined that many of the statistical characteristics of electromagnetic waves scattered from terrain, such as the angular dependence of the mean backscattered power, the range of fading, and the rate of fading, can be duplicated using appropriate targets and acoustical waves in the water. It was found that by examining the leading edge of the received pulses, a mean profile of terrain over the illuminated area may be determined with an error less than 1 part in 300 when using a 6-deg antenna beam from an altitude of $100 \mathrm{~km}$. Warner, Moore, and Edison [1962] reported the use of the simulator in modeling the signal return from over a city. Two model cities were built on a scale of 1 to 500, and the signals from these models were compared with the signal from full-scale radar experiments. The statistical quantity obtained from the acoustic experiment compared favorably with those obtained from the full-scale radar experiment.

The surface roughness of the earth has been reported by Hayre and Moore [1961] and by Hayre [1961]. These papers describe studies designed to determine the validity of the normal bivariate probability distribution and the Gaussian correlation function. Height distribution and correlation studies were also made using various maps of portions of the earth's surface with contour intervals ranging from 1 to $25 \mathrm{ft}$. The results indicated that while the as- 
sumption of normal bivariate distribution is justified, the assumption of Gaussian correlation is not justified. A better correlation seems to be in the form exp $[-|r| \alpha]$, where $r$ is the horizontal distance over which the heights are to be correlated. This results in a different form of scattering cross section which is a function of angle of incidence, surface roughness, and wavelength. This expression is in good agreement with the experimental results. These studies have been extended to take into account the earth-moon distances and provide data on the lunar surfaces. The result as applied to the lunar surfaces will be left to the Commission 5 report.

\subsection{Radiation From the Earth and From Foliage}

Hogg [1962] estimated the brightness temperature of the ground with vegetation at a frequency of 3 $\mathrm{Gc} / \mathrm{s}$ to be that of an almost perfectly absorbing medium; such data are needed to calculate effective antenna noise temperatures. Ring [1962] reported extensive radiometric measurements made at 35 $\mathrm{Gc} / \mathrm{s}$ with the beam pointed toward various surfaces (grass, asphalt, water, concrete) and determined their absorption coefficients versus angle of incidence. Passive measurements of radiation in the centimeter and millimeter range have been made by a number of groups in which they were able to obtain rough maps of the ground which differentiated between surfaces such as water and land. These characteristics varied somewhat with frequency and provided overall types of ground mapping.

Straiton and Tolbert [1963] reported on the radiation at millimeter wavelengths from the earth as seen by a radiometer through an absorbing atmosphere. Curves of the frequency dependence of this radiation were given which showed that when the atmosphere was primarily transparent the radiation was controlled by the ground itself, whereas for the regions where the atmosphere was more opaque, the radiation originated in the atmosphere.

\subsection{Investigation of Transhorizon Radio Wave Propagation over Irregular Terrain}

Research into the propagation of radio waves in the troposphere has been continued by Cornell University over the past several years. During 1960-61 propagation has been studied at $9100 \mathrm{Mc} / \mathrm{s}, 2800$ $\mathrm{Mc} / \mathrm{s}$, and $840 \mathrm{Mc} / \mathrm{s}$ over a $110-\mathrm{km}$ path in the Finger Lakes Region of New York State [Bolgiano, 1961]. Early results of the experiment, which was initiated principally to investigate the wavelength dependence of scatter propagation, indicated surprisingly low values of propagation loss on some occasions and differences between mean loss values measured on spaced receivers that were not readily interpretable in terms of known models. During summer months (June-September), the average propagation loss at all three frequencies frequently decreases by $20 \mathrm{db}$ to $40 \mathrm{db}$ in the early morning hours. The nature of the terrain (pronounced ridges and deep valleys at a 45-deg angle to the path) precludes normal ducting. Detailed field strength measurements made at numerous points along the great circle route between the receiver site and midpath do not support obstacle diffraction theory. An explanation of this phenomenon appears to reside in a meteorological process peculiar to the local terrain. Radiation cooling of high ground after sunset on clear days results in downflow of cool air into the valley bottoms, which contain lakes. This forces the moist air immediately above the lake surfaces to higher altitudes and thus produces elevated layers exhibiting superstandard refractive index gradients. These layers become compressed and further elevated, and the gradients intensified, as the downflow of cooled air continues during the night. By early morning hours they have risen to such altitudes as to have a pronounced effect on this path [Cassam, 1962].

\section{References}

Barsis, A. P., and R. S. Kirby (Sept.-Oct. 1961), VHF and UHF signal characteristics observed on a long knife-edge diffraction path, J. Res. NBS 65D (Radio Prop.), No. 5, 437-448.

Beard, C. I. (Sept. 1961), Coherent and incoherent scattering of microwaves from the ocean, IRE Trans. AP-9, No. 5 , 470-483.

Bolgiano, R., Jr. (Oct. 23-25, 1961), Initial results of a scaled, three-frequency transhorizon propagation experiment, paper presented at URSI Meeting, Austin, Tex.

Cassam, R. P. (1962), An investigation of anomalous transhorizon radio wave propagation over irregular terrain, a paper presented at URSI Meeting, Washington, D.C., May $1-3$.

Edison, A. R., R. K. Moore, and B. D. Warner (May 1960), Radar terrain measured at near-vertical incidence, IRE Trains. AP-8, 3.

Edison, A. R. (Sept. 1961), An acoustic simulator for modeling backscatter of electromagnetic waves, Univ. of New Mexico Report EE-62.

Edison, A. R., R. K. Moore, and B. D. Warner (Apr. 1961), High altitude radio altimeter simulation study, Univ. of New Mexico Technical Rept. EE-53.

Furutsu, K. (Jan.-Feb. 1963), On the theory of radio wave propagation over inhomogeneous earth, J. Res. NBS 69D (Radio Prop.) No. 1, 39-62.

Hayre, H. S., and R. K. Moore (Sept.-Oct. 1961), Theoretical scattering coefficient for near-vertical incidence from contour maps, J. Res NBS 65D (Radio Prop.), No. 5, 427432 .

Hayre, H. S. (Feb. 1961), Roughness and radar return, Proc. IRE 49, No. 2, 223.

Head, Howard T. (June 1960), The influence of trees on television field strengths at ultra-high frequencies, Proc. IRE 48, 6 .

Hogg, D. C. (1962), A study of the sources of noise in centimeter wave antenna, Low Noise Electronics (Pergamon Press, Oxford).

Katz, I., and L. M. Spetner (1960), Polarization and depression-angle dependence of radar terrain return, J. Res. NBS 64D (Radio Prop.), No. 5, 483.

Katz, I. (June 1962), Radar reflectivity of the ocean surface for circular polarization, APL/JHU Internal Memo BPD62U-4, IRE Trans. PGAP, in press.

LaGrone, A. H. (June 1960), Forecasting television service fields, Proc. IRE 48, 6.

LaGrone, A. H., and C. W. Chapman (Sept. 1961), Some propagation characteristics of high UHF signals in the immediate vicinity of trees, IRE Trans. AP-9.

LaGrone, A. H., P. E. Martin, and C. W. Chapman (1963), Height-gain measurements at VHF and UHF behind a grove of trees, IRE Trans. Broadcasting, in press. 
Nielson, D., G. Hagn, L. Rorden, and N. Clark (May 1960), An investigation of the backscatter of high frequency radio waves from land, sea, water, and ice, Stanford Res. Inst. Final Report.

Ransone, J. T., W. T. Davis, and J. C. Daley (1962), Radar echoes from ships, NRL memo report No. 1368, Oct. 30.

Ring, D. H. (1962), Report AF-19-(122)-458 to Lincoln Laboratories 1956, released in 1962.

Schooley, A. H. (Apr. 1962), Upwind-downwind ration of radar return calculated from facet size statistics of a winddisturbed water surface, Proc. IRE 50, Pt. I, 456-461.
Straiton, A. W., and C. W. Tolbert, Factors affecting earth satellite millimeter communication, IRE Trans. MTT (submitted).

Wait, J. R., and A. M. Conda (1959), Diffraction of electromagnetic waves by smooth obstacles for grazing angles, J. Res. NBS 63D (Radio Prop.), No. 5, 181-197.

Waite, A. H., and S. J. Schmidt (June 1962), Gross errors in height indication from pulsed radar altimeters operating over thick ice or snow, Proc. IRE 50, No. 6, 1515-1520.

Warner, B. D., R. K. Moore, and A. R. Edison (July 1962), Acoustic simulation of radar altimeter signals from cities, University of New Mexico Report EE-77.

\section{Guided Waves in the Troposphere}

\subsection{Tropospheric Layers}

With the advent of satellites, renewed interest has developed in the phenomenon of radio wave guiding by the earth's atmosphere. Anderson [1961] has reported the reception of satellite signals at $108 \mathrm{Mc} / \mathrm{s}$ and $212 \mathrm{Mc} / \mathrm{s}$ minutes before the satellite rises above the horizon over the Pacific. On one occasion reception was obtained at a range $2,000 \mathrm{mi}$ beyond the radio horizon. Arendt and Soicher [1962] have observed similar extensions of the radio horizon which they also attribute to guiding in the troposphere.

In regions where the earth's atmosphere subsides, especially over water, there are large areas characterized by a layer of moist, cool air adjacent to the earth and covered by a warm, dry air mass. The increase in temperature and decrease in the amount of water vapor with elevation through the transition region between these air masses produce a rapid decrease in refractive index, thus forming a radio duct. An analysis by Katzin et al. [1960] of radiosonde and airborne refractometer data from the South Atlantic trade-wind regions indicated the presence of a duct practically all of the time. Subsequent radio transmission measurements at $220 \mathrm{Mc} / \mathrm{s}$ by Ringwalt and Macdonald [1961] showed that greatly increased radio ranges were possible under such conditions. The fields decreased with distance at a rate ranging from $6 \mathrm{db}$ per 100 nautical miles to the so-called "scatter" level of about $15 \mathrm{db}$ per 100 nautical miles; maximum ranges of 500 to $1,200 \mathrm{mi}$ were observed. The variation of signal level with elevation was quite striking; the fields were almost always maximum in the transition layer, being some $25 \mathrm{db}$ greater than the average at lower levels.

Results of a similar propagation experiment at 128 $\mathrm{Mc} / \mathrm{s}$ over the airline route from San Francisco to Honolulu showed aircraft-ground transmission losses only 20 to $40 \mathrm{db}$ in excess of free space loss out to ranges of 2,200 $\mathrm{mi}$ for aircraft altitudes of 28,000 to $35,000 \mathrm{ft}$ [Vergara, Levatich, and Carroll, 1962]. The enhanced radio ranges were found to be correlated with a proposed meteorological parameter related to mean index gradient and atmospheric stability. Enhanced radio ranges have also been observed at 220 and $445 \mathrm{Mc} / \mathrm{s}$ in the Pacific between San Diego and Oahu [Randall, 1962]. Signals only
$20 \mathrm{db}$ below free space were measured to ranges of 1,800 nautical miles.

Ament [1961] has discussed the role of refractivity irregularities in ducts and concluded that the observed correlations among duct strengths, attenuation rates, and height gains in and above the duct can be explained in terms of normal duct theory modified to account for the scattering caused by the irregularities.

\subsection{Ground Wave and VLF Propagation}

Propagation of VLF waves between the boundaries of the earth and the ionosphere may be considered as guided waves in the troposphere. Wait [1962a, b, and $c]$ has investigated the excitation of VLF modes and their propagation characteristics for a variable ionospheric height. He has also investigated the effects of a gradual transition at the boundary between the non-ionized lower atmosphere and the ionosphere. Crain and Tamarkin [1961] have reported sudden changes of VLF propagation characteristics caused by a high-altitude nuclear detonation which they attribute to bomb-induced ionization in the ionospheric $D$ layer.

Johler and Lilley [1961] have analyzed sferic signatures of thunderstorms at low radiofrequencies for determining the conductivity of the ground.

The nature of radiowave propagation through the earth (and sea) and at the earth-atmosphere (and sea-atmosphere) interface has been examined extensively by Commission 2 . It was the subject of two sessions organized by J.R. Wait at the spring URSI meeting in Washington, D.C., May 1962. Wait has also organized a collection of papers on surface and subsurface propagation being published in a special issue of the Transactions of the IEEE on Antennas and Propagation (AP-11, No. 3, 1963). This area represents an overlap of the interests of Commissions 2 and 6 . To avoid repetition, this material is included in the section prepared by Commission 6 under the heading Electromagnetic Fields in Lossy Media.

\section{References}

Ament, W. S. (1961), Transhorizon propagation in stratified scattering atmosphere, a paper presented at Fall URSI Meeting, Austin. 
Anderson, L. J. (May 20, 1961), 108-216 Mc/s radio signals from satellites below the horizon, Nature $\mathbf{1 9 0}$.

Arendt, P. R., and H. Soicher (Oct. 1962), Radio horizon distribution variation on $108 \mathrm{Mc}$ measured with satellite signals, URSI Ottawa Meeting.

Crain, C. M., and P. Tamarkin (1961), A note on the cause of sudden ionization anomalies in regions remote from high altitude bursts, J. Geophys, Res. 66, 35-39.

Johler, J. R., and C. M. Lilley (1962), Ground-conductivity determinations by an analysis of the sferic signatures of thunderstorms, J. Geophys. Res. 66, 3823-3828.

Katzin, M., H. Pezzner, B. Y. C. Koo, J. V. Larson, and J. C. Katzin (May-June 1960), The trade-wind inversion as a transoceanic duct. J. Res. NBS 64D (Radio Prop.), No. 3, 247-253.

Randall, D. L. (May 1962), Project Tradewinds IV: Radiometeorological conditions in the elevated duct between San Diego and Honolulu (June 22 and 26, 1961), Reprint from NRL Progress Report.

Ringwalt, D. L., and F. C. Macdonald (July 1961), Elevated duct propagation in the tradewinds, IRE Trans. AP-9, 4, $377-383$.

Vergara, W. C., J. H. Levatich, and T. J. Carroll (Sept. 1962), VHF air-ground propagation far beyond the horizon and tropospheric stability. IRE Trans. AP-10, No. 5, 608621.

Wait, J. R. (1962a), Excitation of modes at low frequency in the earth-ionosphere wave guide, J. Geophys. Res. 67, $3823-3828$

Wait, J. R. (1962b), An analysis of VLF mode propagation for a variable ionosphere height, J. Res. NBS $66 \mathbf{D}$ (Radio Prop.), No. 4, 453-461.

Wait, J. R. (1962c), On the propagation of VLF and ELF radio waves when the ionosphere is not sharply bounded, J. Res. NBS 66D (Radio Prop.), No. 1, 53-61.

Anderson, W. L., and R. K. Moore (1960), Frequency spectra of transient electromagnetic pulses in a conducting medium, IRE Trans. AP-8, 603 .

Burrows, C. R. (1962), D.C. signaling in conducting media, IRE Trans. AP-10, $328-334$.

Burrows, C. R. (1963), Transient response in an imperfect dielectric (to be published in Special PGAP Issue on Subsurface Propagation).

de Bettencourt, J. T., and J. Carolan (1963), Radio propagation in deep strata along rock-overburden interface (to be published in Special PGAP Issue on Sub-surface Propagation).

Durrani, S. H. (1962), Air-to-undersea communication, Proc. IRE 50, 96-97.

Durrani, S. H. (1962), Air-to-undersea communication with electric dipoles, IRE Trans. AP-10, No. 5, 524-528.

Galejs, J. (1960), Impulse excitation of a conducting medium, IRE Trans. AP-8, 227-228.

Gerks, I. H. (1962), Use of a high-speed computer for ground wave calculations, IRE Trans. AP-10, 292-299.

Ghose, R. N. (1960), The long range sub-surface communication system, Proc. Sixth IRE Nat. Comm. Symp., 110-120.

Hansen, R. C. (1963), Radiation and reception with buried and submerged antennas (to be published in Special PGAP Issue on Sub-surface Propagation).

Harmon, G. J. (1961), Radio wave propagation through the earth's rock strata - a new medium of communication, IRE Globecom Conv. Rec., 31-34.

Jean, A. G., A. C. Murphy, J. R. Wait, and D. F. Wasmundt (Sept-Oct. 1961), Observed attenuation rate of ELF radio waves, J. Res. NBS 65D (Radio Prop.), No. 5, 475-479.

Johler, J. R. (1962), Propagation of the low frequency radio signal, Proc. IRE 50, 404-427.

Keller, G. V. (1963), Electrical properties in the deep crust (to be published in Special PGAP Issue on Sub-surface Propagation).
Logan, N. A. (1962), A mathematical model for diffraction by convex surfaces, in Electromagnetic Waves (ed. R. E. Langer), Univ. of Wisconsin Press, 139-180.

Mijnarends, P. E. (1962), Propagation of electromagnetic step functions over conducting media, J. Appl. Phys. 33, 2556-2564.

Vogler, L. (1963), Point-to-point communication on the moon, J. Res. NBS 67D (Radio Prop.), No. 1, 5-21.

Wait, J. R. (Sept. 1960), A conference on the propagation of ELF electromagnetic waves, Proc. IRE 48, No. 9, 1648.

Wait, J. R. (July, 1960), Influence of source distance on the impedance characteristics of ELF radio waves, Proc. IRE 48, No. 7, 1338 .

Wait, J. R. (ed.) (June 6, 1960), Proceedings of the 1960 Conference on the propagation of ELF radio waves, NBS Tech. Note 61 (PB161562).

Wait, J. R. (1960), Propagation of electromagnetic pulses in a homogeneous conducting earth, Appl. Sci. Res. B8, $213-253$

Wait, J. R. (Jan.-Feb. 1961), A new approach to the mode theory of VLF propagation, J. Res. NBS 65D (Radio Prop.), No. 1, 37-46.

Wait, J.'R. (June 1961), A comparison between theoretical and experimental data on phase velocity of VLF radio waves, Proc. IRE 49, No. 6, 1089-1090.

Wait, J. R. (Sept-Oct. 1961), A note concerning the excitation of ELF electromagnetic waves, J. Res. NBS 65D (Radio Prop.), No. 5, 481-484.

Wait, J. R. (Sept. 1961), On the possibility of rejecting certain modes in VLF propagation, Proc. IRE 49, No. 9, 1429

Wait, J. R. (July-Aug. 1961), On the theory of mixed-path ground wave propagation on a spherical earth, J. Res. NBS 64D (Radio Prop.), No. 4, 401-410.

W ait, J. R. (1961), The electromagnetic fields of a horizontal dipole in the presence of a conducting half-space, Can. J. Phys. 39, 1017-1028.

Wait, J. R. (Jan. 1962), Average decay laws for VLF fields, Proc. IRE 50, No. 1, 53-56.

Wait, J. R. (July 1962), Introduction to the theory of VLF Propagation, Proc. IRE 50, 1624-1647.

Wait, J. R. (1962), Electromagnetic Waves in Stratified Media, Pergamon Press, Oxford.

Wait, J. R. (1962), A note on the propagation of electromagnetic pulses over the earth's surface, Can. J. Phys. 40, $1264-1268$.

Wait, J. R. (1962), The propagation of electromagnetic waves along the earth's surface, in Electromagnetic Waves (ed. R. E. Langer), Univ. of Wisconsin Press, 243-290.

Wait, J. R., A note on diurnal phase changes of VLF waves for long paths (to be published in January $1963 \mathrm{~J}$. Geophys. Res.).

Wait, J. R., and Nancy F. Carter (Mar. 1960), Field strength calculations for ELF radio waves, NBS Tech. Note. 52.

Wait, J. R., and K. Spies (Aug. 1960), Influence of earth curvature and the terrestrial magnetic field on VLF propagation, J. Geophys. Res. 65, No. 8, 2325-2331.

Wait, J. R., and K. P. Spies (Mar. 1961), A note on the phase velocity of VLF radio waves, J. Geophys. Res. 66. No. 3, 992-993.

Wait, J. R., and L. C. Walters (1963), Curves for ground wave propagation over mixed land and sea paths, IRE Trans. PGAP (in press).

Walters, L. C., and J. R. Johler (Jan.-Feb. 1962), On the diffraction of spherical radio waves by a finitely conducting spherical earth, J. Res. NBS 66D (Radio Prop.), 101-106.

Wheeler, H. A. (1961), Radio wave propagation in the earth's crust, J. Res. NBS 65D (Radio Prop.), 189-191.

Zisk, S. H. (1960), Electromagnetic transients in conducting media, IRE Trans. AP-8, 229-230. 


\section{Radar Studies of the Sun, Moon, and Planets}

The growth in sensitivity and distribution of large radar systems over the past several years has greatly stimulated the study of objects in the solar system by means of radio echoes. 'To date only the Sun, the Moon, and Venus have been successfully studied, but it is expected that Mercury and Mars will shortly be added to the list of accessible targets.

\subsection{The Sun}

The possibility of obtaining radar echoes from the sun was suggested first by Kerr [1952] and later reexamined by Bass and Braude [1957]. Both papers were essentially in agreement in estimating the cross section of the sun to be approximately $1.5 \pi R_{0}^{2}$ at $30 \mathrm{Mc} / \mathrm{s}$ and in estimating the magnitude of the radar system required to obtain echoes at this frequency.

Radar contact with the sun was first established in 1958-59 when Eshleman, Barthle, and Gallagher [1960] obtained echoes at $26 \mathrm{Mc} / \mathrm{s}$. Their experiments were confined to several days during the equinoctial periods when the sun traversed the beam of a large fixed broadside array of rhombic antennas. The processed data showed unmistakable evidence of peak correlation with very low error probability corresponding to radar reflections from regions in the solar corona near two solar radii [Barthle, 1960]. Based upon the detection thresholds obtained by these techniques, it would appear that the effective radar cross section of the sun at $26 \mathrm{Mc} / \mathrm{s}$ was as large as or greater than the value of $1.5 \pi R_{0}^{2}$ estimated by Kerr and by Bass and Braude.

In the early summer of 1961, Massachusetts Institute of Technology Lincoln Laboratory began a regular daily series of radar observations of the solar corona at $38 \mathrm{Mc} / \mathrm{s}$ utilizing a large phased array antenna and powerful radar system located near El Campo, Tex. $\left(29.00^{\circ} \mathrm{N}\right.$ latitude and $96.25^{\circ} \mathrm{W}$ longitude). The antenna system consists of 1024 dipoles arrayed in a north-south direction in eight (8) rows of 128 elements. This antenna has a maximum effective gain of $34 \mathrm{db}$ (isotropic) and produces a fan beam $0.8 \times 12 \mathrm{deg}$. Each radiating element is equipped with phasing cables which allow the fan beam to be positioned to the declination of the sun throughout the year. The transmitter is operated at $500 \mathrm{kw} \mathrm{CW}$. The radar system is equipped with correlation and integration devices that permit unambiguous range measurements of weak signals well below the background solar noise [Abel, Chisholm, Fleck, and James, 1962].

A new solar radar system was installed at Stanford University in 1961, consisting of an antenna array of $48 \mathrm{log}$-periodic elements together with appropriate phasing and tuning devices to permit radar operation at a desired frequency in a $20-$ to $60-\mathrm{Mc} / \mathrm{s}$ band and a $300-\mathrm{kw}$ average power transmitter. The transmitter may also be used with an adjacent $150-\mathrm{ft}$ diam steerable paraboloidal antenna. Preliminary operation of this system for solar studies was unfortunately delayed until September-October 1962, and specific results are not available at this date pending completion of the processing of the data obtained during these measurements.

Several other large radar systems which are potentially applicable to radar studies of the solar corona either have been completed or are nearing completion. The National Bureau of Standards has built a powerful 50-Mc/s radar system located at the geomagnetic equator in Peru for studies of incoherent electron scatter in the ionosphere. The antenna consists of 9,000 orthogonally polarized dipole elements covering a $1,000-\mathrm{ft}$ sq horizontal area. The ultimate transmitter power for this system is 400 to $800 \mathrm{kw}$ average with a $6-\mathrm{Mw}$ peak power at pulse widths up to $2 \mathrm{~m}$ in length. Some solar radar studies are planned during the periods of the year when the sun will traverse the narrow vertical beam of the radar system.

The initial results of the MIT Lincoln Laboratory solar observations in the summer of 1961 indicate considerable daily variability in the strength of the solar echoes and in apparent coronal cross section. The signals detected in a $2-\mathrm{kc} / \mathrm{s}$ bandwidth at 38 $\mathrm{Mc} / \mathrm{s}$ were substantially lower $(10$ to $15 \mathrm{db})$ than those detected by Eshleman, Barthle, and Gallagher in 1958-1959 at $26 \mathrm{Mc} / \mathrm{s}$ also utilizing a $2-\mathrm{kc} / \mathrm{s}$ bandwidth [Abel, Chisholm, Fleck, and James, 1961]. Later the receiving bandwidths were progressively widened to $4 \mathrm{kc} / \mathrm{s}$, then to 8 and finally $16 \mathrm{kc} / \mathrm{s}$. One of the most significant results obtained from these investigations is the evidence that the Doppler broadening apparently caused by internal motion in the corona exceeds the Doppler broadening expected from solar rotation alone $(\sim 1.5 \mathrm{kc} / \mathrm{s}$ at $38 \mathrm{Mc} / \mathrm{s})$ by at least a factor of 10 with some recent evidence of Doppler broadened returns over a $40-\mathrm{kc} / \mathrm{s}$ band [Abel, Chisholm, and James, 1962]. In September 1961, the solar radar system was equipped with a broad-band magnetic tape recording facility which allowed systematic studies of the Doppler broadened spectrum in $1-\mathrm{kc} / \mathrm{s}$ intervals over a $16-\mathrm{kc} / \mathrm{s}$ band. These Doppler spectra indicate that the maximum energy density occurs at Doppler frequencies 3 to $4 \mathrm{kc} / \mathrm{s}$ higher than the carrier frequency, decreasing on either side to about one-half the energy density at about $8 \mathrm{kc} / \mathrm{s}$ from the maximum value. These data suggest a mean outward motion of 12 to 16 $\mathrm{km} / \mathrm{sec}$ in the coronal regions producing the radar echoes accompanied by chaotic motions of an ensemble of scatterers having mean velocities in the region of $30 \mathrm{~km} / \mathrm{sec}$ or greater.

Measurements of the range of the solar echoes at $38 \mathrm{Mc} / \mathrm{s}$ indicate radar returns distributed over at least 5 sec in time of arrival corresponding to a scattering region extending over a range of $7.5 \times 10^{5}$ $\mathrm{km}$. The maximum echo strength occurs near 1.5 solar radii from the center of the sun towards the earth. This range was computed assuming $1.6 \mathrm{sec}$ 
of additional group delay for a $38-\mathrm{Mc} / \mathrm{s}$ signal entering and emerging from the turning point of the central ray in the corona [Chisholm, 1960]. The apparent range of the maximum solar radar returns varies over a range of 1 to 2 sec from day to day.

A measure of the variability of the radar cross section of the solar corona over a yearly period has been obtained by summing the daily radar returns in an $8-\mathrm{kc} / \mathrm{s}$ bandwidth over all detectable ranges for comparison with other radio and optical indices of solar activity. The average radar cross section derived from these data does not give the total cross section since some fraction of the radar returns are known to be Doppler shifted out of this bandwidth. Calculated on the basis of this bandwidth the average cross section is about one-fourth the projected area of the photosphere $\left(\pi R_{0}^{2}\right)$. The trend of the daily variations of the cross section appears to have a good correlation with the rotational period of the sun. The day-to-day correlation of the radar cross section with daily flare and solar radio noise activity is as yet inconclusive. Further detailed study is under way to compare this activity for specific time intervals of the radar operation.

Operation of the El Campo Solar Radar system will be continued in 1963 on a regular daily schedule. The system has recently been modified for operation with a bandwidth as wide as $50-\mathrm{kc} / \mathrm{s}$ and to permit Doppler measurements over this range. It is further planned to double the size of the array in early 1963 to provide additional sensitivity.

The advances in powerful radar systems and in signal processing techniques in the United States in the last few years appear to have demonstrated the feasibility of radar methods for studies of the structure of the solar corona and to hold future promise of radar measurements over a relatively broad range of frequencies as more powerful radar systems are placed in operation with their full designed power capabilities.

\subsection{The Moon}

Prior to 1960, Trexler [1958] in the United States and Evans [1957] in England had shown that the moon acts in large part as a smooth reflector of incident radio energy. This result, in striking contrast to the situation at optical wavelengths, implies that a large fraction of the lunar surface must be relatively smooth on the macroscopic scale explored at radio wavelengths.

Measurements which began in 1960 [Leadabrand et al., 1960; Pettengill, 1960; Hughes, 1960] have extended the earlier work and, by using relatively short pulses to explore the detailed time distribution of the reflected power, have permitted a quantitative description of the mean lunar slopes, which are found to have a mean gradient of about 1 in 10 [Daniels, 1961; Hagfors, 1961; Hughes, 1961]. Echoes have been detected from all portions of the lunar surface, even from the limbs. An analysis of the angular scattering behavior and degree of polarization of the lunar echoes appears to require that a portion of the surface approximately 8 percent on the average for measurements at 68 -cm wavelength, scatter diffusely [Pettengill and Henry, 1962a].

More recent measurements by Evans and Pettengill [1963] at wavelengths substantially shorter $(3.6 \mathrm{~cm})$ and longer $(7.8 \mathrm{~m})$ than previous work indicate that the transition of the moon from a largely specular scatterer at meter wavelengths to a completely diffuse scatterer at optical wavelengths is a gradual process. Specifically, the mean gradient is found to vary with wavelengths being about one in 11 for points spaced by $68 \mathrm{~cm}$ and one in seven for points at $3.6 \mathrm{~cm}$. Similarly, at $68 \mathrm{~cm}$ about 8 percent of the surface appears to be covered with structure of the order of a wavelength in size, whereas at $3.6 \mathrm{~cm}$ the corresponding figure is 14 percent.

The most precise measurements of lunar radar cross section to date find a value of 0.074 of the projected area of the moon at $73 \mathrm{~cm}$ [Fricker et al., 1960]. Equally precise values at other wavelengths do not yet appear to have been obtained, although there is some evidence for a gradual reduction with decreasing wavelength. The cross section measured at $73 \mathrm{~cm}$ corresponds to a near-surface dielectric constant of 2.8, or about half that for typical rocks of the earth's surface. It is speculated that the low lunar value probably results from a high surface porosity.

An experimental technique for determining radar scattering properties of relatively small regions of the lunar surface was described and its general feasibility shown in early 1960. More recently this method has been applied to a study of the crater Tycho, which has been shown to be an area of unusual roughness as compared to the average lunar surface [Pettengill and Henry, 1962b].

\subsection{The Planet Venus}

The first radio echoes from the planet Venus appear to have been obtained during the inferior conjunction which occurred in the spring of 1961. Five groups of workers, three in the United States, one in the United Kingdom, and one in the U.S.S.R., were successful in their attempts at that time, The U.S. groups were at Millstone, M.I.T. [Pettengill et al., 1962c], Goldstone, J.P.L. [Muhleman, Holdridge, and Block, 1962], and Moorestown, R.C.A. [Maron, Luchak, and Blitzstein, 1961]. The U.K. group was at Jodrell Bank [Thomson et al., 1961]. Results for the U.S.S.R. group were reported by Kotei'nikov et al. [1962]. A summary of the results as reflected in a determination of the value of the astronomical unit is given in table 1 . The accuracy

TABLE 1

\begin{tabular}{|c|c|c|}
\hline Facility & $\begin{array}{l}\text { Radar } \\
\text { frequency }\end{array}$ & Astronomical unit \\
\hline $\begin{array}{l}\text { Millstone (M.I.T.) } \\
\text { Goldstone (J.P.L.) } \\
\text { Jodrell Bank (U. of Manchester) } \\
\text { Moorestown, N.J. (R.C.A.) } \\
\text { U.S.S.R }\end{array}$ & $\begin{array}{r}(M c s) \\
440 \\
2388 \\
408 \\
700\end{array}$ & $\begin{array}{l}\quad \mathrm{km} \\
149,597,850 \pm 400 \\
149.598 .600^{*} \pm 250 \\
149,601,000 \pm 5000 \\
149,596,000 \text { (no error given) } \\
149,599,500 \pm 800\end{array}$ \\
\hline
\end{tabular}

*The result quoted in the reference given has been here modified to correspond to a light velocity: $c=299,792.5 \mathrm{~km} / \mathrm{sec}$ (as used by the other groups). 
obtained, even in the worst case, far exceeded that previously available from optical observations alone.

The Millstone and Goldstone groups succeeded in making measurements over a sufficiently long interval to find additional systematic errors which were attributed to inaccuracies in the orbits of Venus and earth. These investigators were also able to show that the reflectivity of Venus was near 0.1 and did not vary appreciably over the wavelength interval from $12.5 \mathrm{~cm}$ to $68 \mathrm{~cm}$, thus ruling out a Cytherean ionosphere as the source of the echoes. The measured range dispersion suggested a surface smoother than that observed for the moon. These measurements, when combined with the narrow returned frequency spectrum, require a very long period of planetary rotation (in excess of 100 days, probably synchronous).

\section{References}

Abel, W. G., J. H. Chisholm, P. L. Fleck, and J. C. James (Dec. 1961), Radar reflections from the sun at very high frequencies, J. Geophys. Res. 66, 4303-4307.

Abel, W. G., J. H. Chisholm, P. L. Fleck, and J. C. James (1962), A VHF solar radar system, Part 5, IRE International Conv. Rec. New York, pp. 58-66.

Abel, W. G., J. H. Chisholm, and J. C. James (May 1962), Radar reflections from the sun at VHF, Paper presented at the Third International Space Science Symposium (COSPAR), Washington, D.C. Scheduled for publication in SPACE Research III, Proceedings of the Third Space Science Symposium (North Holland Publ. Co.).

Barthle, R. C. (Aug. 24, 1960), The detection of radar echoes from the sun, Stanford Electronics Laboratories, Stanford University Scientific Rept. No. 9.

Bass, F. G., and S. I. Braude (1957), On the question of reflecting radar signals from the sun, Ukrainian J. Phys. 2, 149-168.

Chisholm, J. H. (1960), Some physical aspects of radar probing of the sun's atmosphere, Notes for 1960 summer radar astronomy course at Mass. Institute of Technology, Scheduled for publication in Radar Astronomy (McGrawHill Publishing Co., New York, N.Y.).

Daniels, F. B. (1961), A theory of radar reflection from the moon and planets, J. Geophys. Res. 66, 1781-1788.

Eshleman, V. R., R. C. Barthle, and P. B. Gallagher (Feb. 5, 1960), Radar echoes from the sun, Science 131, 3397, 329-332.
Evans, J. V. (1957), The scattering of radio waves by the moon, Proc. Phys. Soc. B70, 1105-1112.

Evans, J. V., and G. H. Pettengill (Jan. 1963), The scattering behavior of the moon at wavelengths of $3.6,68$ and 784 cm, J. Geophys. Res. 68, 423-447.

Fricker, S. J., R. P. Ingalls, W. E. Mason, M. L. Stone, and D. W. Swift (1960), Computation and measurement of the fading rate of moon reflected UHF signals, J. Res. NBS 64D (Radio Prop.), No. 5, 455-465.

Hagfors, T. (1961), Some properties of radio waves reflected from the moon and their relation to the lunar surface, J. Geophys. Res. 66, 777-785.

Hatanaka, Takeo (Oct. 15, 1959), Reflection of radio waves from the sun, Research Report EE 450, School of Electrical Engineering, Cornell University.

Hughes, V. A. (1960), Roughness of the moon as a radar reflector, Nature 186, 873-874.

Hughes, V. A. (1961), Radio wave scattering from the lunar surface, Proc. Phys. Soc. 78, 988-997.

Kerr, F. J. (June 1952), On the possibility of obtaining radar echoes from the sun and planets, Proc. IRE 40, 660-666.

Kotel'nikov, V. A., et al., (1962), Radar observation of Venus, Doklady Ákad. Nauk 145, 1035-1038.

Leadabrand, R. L., R. B. Dyce, A. Fredriksen, R. I. Presnell, and R. C. Barthle (1960), Evidence that the moon is a rough scatterer at radio frequencies, J. Geophys. Res. 65, 3071-3078.

Maron, I., G. Luchak, and W. Blitzstein (1961), Radar observation of Venus, Science 134, 1419-1421.

Muhleman, D. O., D. B. Holdridge, and N. Block (1962), The astronomical unit determined by radar reflections from Venus, Astron. J. 6\%, 191-203.

Pettengill, G. H. (1960), Measurements of lunar reflectivity using the millstone radar, Proc. IRE 48, 933-934.

Pettengill, G. H., and J. C. Henry (1962a), Radar measurements of the lunar surface, The Moon, I.A.U. Symposium No. 14, ed. Z. Kopal and Z. Mikhailov (Academic Press, London).

Pettengill, G. H., and J. C. Henry (1962b), Enhancement of radar reflectivity associated with the lunar crater Tycho, J. Geophys. Res. 67, 4881-4885.

Pettengill, G. H., H. W. Briscoe, J. V. Evans, E. Gehrels, G. M. Hyde, L. G. Kraft, R. Price, and W. B. Smith (1962c), Radar investigation of Venus, Astron. J. 67, $181-190$

Thomson, J. H., G. N. Taylor, J. E. B. Ponsonby, and R. S. Roger (1961), A new determination of the solar parallax by means of radar echoes from Venus, Nature 190, 519-520.

Trexler, J. H. (1958), Lunar radio echoes, Proc. IRE 46, $256-292$.

(Paper 68D5-361) 